NBER WORKING PAPER SERIES

\title{
LINEAR SOCIAL INTERACTIONS MODELS
}

Lawrence E. Blume

William A. Brock

Steven N. Durlauf

Rajshri Jayaraman

Working Paper 19212

http://www.nber.org/papers/w19212

\author{
NATIONAL BUREAU OF ECONOMIC RESEARCH \\ 1050 Massachusetts Avenue \\ Cambridge, MA 02138 \\ July 2013
}

Support of the Human Capital and Economic Opportunity Global Working Group, sponsored by the Institute for New Economic Thinking, is gratefully acknowledged by all authors. Financial support has been supplied to Blume by NSF grant CCF-0910940, WWTF Grant "Die Evolution von Normen und Konventionen in der Wirtschaft", and ARO MURI Award No. W911NF-12-1-0509, to Brock by the Vilas Trust, and to Durlauf by the University of Wisconsin Graduate School, the Laurits Christensen Chair in Economics, and the Vilas Trust, all of which is greatly appreciated. We thank the editor and three referees for very helpful comments and suggestions. Wallice Ao, Joel Han, Hon Ho Kwok, Ariel Roginsky, Kegon Tan and Xiangrong Yu have provided superb research assistance. We are grateful for comments from James Heckman, Youcef Msaid, Debraj Ray, Alex Rees-Jones, Dean Robinson, Michael Strain, and Nichole Szembrot; and to George Jakubson and Charles Manski for discussions of the issues we address. This paper was written in honor of James J. Heckman, whose influence will be evident throughout. The views expressed herein are those of the authors and do not necessarily reflect the views of the National Bureau of Economic Research.

NBER working papers are circulated for discussion and comment purposes. They have not been peerreviewed or been subject to the review by the NBER Board of Directors that accompanies official NBER publications.

(C) 2013 by Lawrence E. Blume, William A. Brock, Steven N. Durlauf, and Rajshri Jayaraman. All rights reserved. Short sections of text, not to exceed two paragraphs, may be quoted without explicit permission provided that full credit, including (C) notice, is given to the source. 
Linear Social Interactions Models

Lawrence E. Blume, William A. Brock, Steven N. Durlauf, and Rajshri Jayaraman

NBER Working Paper No. 19212

July 2013

JEL No. C21,C23,C31,C35,C72,Z13

\begin{abstract}
$\underline{\text { ABSTRACT }}$
This paper provides a systematic analysis of identification in linear social interactions models. This is both a theoretical and an econometric exercise as the analysis is linked to a rigorously delineated model of interdependent decisions. We develop an incomplete information game that describes individual choices in the presence of social interactions. The equilibrium strategy profiles are linear. Standard models in the empirical social interactions literature are shown to be exact or approximate special cases of our general framework, which in turn provides a basis for understanding the microeconomic foundations of those models. We consider identification of both endogenous (peer) and contextual social effects under alternative assumptions on a priori information about network structure available to an analyst, and contrast the informational content of individual-level and aggregated data. Finally, we discuss potential ramifications for identification of endogenous group selection and differences between the information sets of analysts and agents.
\end{abstract}

Lawrence E. Blume

Department of Economics

Uris Hall

Cornell University

Ithaca, NY 14850

1b19@cornell.edu

William A. Brock

Department of Economics

University of Wisconsin

1180 Observatory Drive

Madison, WI 537061393

wbrock@ssc.wisc.edu

\author{
Steven N. Durlauf \\ Department of Economics \\ University of Wisconsin \\ 1180 Observatory Drive \\ Madison, WI 53706-1393 \\ and NBER \\ sdurlauf@ssc.wisc.edu \\ Rajshri Jayaraman \\ European School of Management \\ and Technology ESMT \\ Schlossplatz 1 \\ 10178 Berlin \\ Germany \\ jayaraman@esmt.org
}


...it is said by some that men will think and act for themselves; that none will disuse spirits or anything else, merely because his neighbors do; and that moral influence is not the powerful engine contended for...Let me ask the man who would maintain this position most stiffly, what compensation he will accept to go to church some Sunday and sit during the sermon with his wife's bonnet upon his head? Not a trifle, l'll venture. There would be nothing irreligious in it...Then why not? Is it not because there would be something egregiously unfashionable about it? Then it is the influence of fashion; and what is the influence of fashion but the influence that other people's actions have on our own actions, the strong inclination each of us feels to do as we see our neighbors do? Nor is the influence of fashion confined to any particular thing or class of things. It is just as strong on one subject as another.

Abraham Lincoln,

February 22, 1842

Address to the Washington Temperance Society of Springfield Illinois

\section{INTRODUCTION}

While the proposition that individuals are subject to social influence states the obvious, the study of social influences on individual behavior in economics is a relatively recent phenomenon. ${ }^{1}$ In the last two decades, however, a rich theoretical, econometric, and empirical literature in social economics, comprehensively overviewed in Benhabib, Bisin, and Jackson (2011a,b), has emerged. While each of these three dimensions - the theoretical, econometric, and empirical - has made important advances, it is fair to say that they are yet to be well integrated. By this we mean that the theoretical models used to study social interactions are distinct from the econometric environments in which identification is studied, while empirical work generally does not systematically exploit the implications of theory and econometrics for the formulation of data analyses.

The objective of this paper is to facilitate the integration of the theoretical, econometric, and empirical sides of the social interactions literature through a systematic investigation of linear social interactions models. Linear models are the workhorse of empirical research and have been the primary subject of econometric work on the identification of social interactions since Manski (1993). Our analysis provides rigorous microfoundations for a broad class of linear social interactions models.

The central, and fundamentally optimistic, message of this paper is that in most cases, linear social interactions models are identified. We employ a theoretically

\footnotetext{
${ }^{1}$ Of course there are exceptions to this claim. Becker (1974) is an example of theoretical analysis that predate the modern literature, and Henderson, Mieszkowski, and Sauvageau (1978) and Datcher (1982) are early and seminal contributions in the empirical study of neighborhood effects. Examples of recent empirical applications of social interactions models include Conley and Udry (2010) on the diffusion of technology; Nakajima (2007) on smoking; Sirakaya (2006) on crime; Rege, Telle, and Votruba (2012) on the take up of welfare programs; and Topa (2001) and Bayer, Ross, and Topa (2008) on labor market outcomes. Another major area of social interactions work is education, which we discuss throughout the paper.
} 
grounded model to understand the conditions under which social interaction effects are or are not identified. The identification problem is shown to depend on three factors: the prior knowledge available to an analyst on the social structure characterizing direct interactions between individuals, the type of data available to the analyst - whether aggregated or individual level - and the implications of endogenous network formation for the conditional expectations of unobserved heterogeneity given the social structure. The onus on the empiricist lies in establishing what they know about social networks a priori and, conditional on this information, verifying that their social interactions model satisfies the conditions needed for identification, many of which are provided in this paper. The conditions we describe do not involve adding stronger assumptions than have appeared in previous papers. Rather, we show that in some cases, non-identification results are artifices of strong assumptions and in others, we establish identification under weaker assumptions than have been previously employed.

We start by providing rigorous microfoundations that either exactly nest or approximate the many linear econometric models that have appeared in the social interactions literature. This is useful for empiricists because it permits a structural interpretation of regression parameter estimates, thereby allowing particular studies to shed light on more general contexts. Further, these microfoundations allow one to assess whether particular regression formulations that have appeared in various econometric and empirical studies are sensible when one considers them as equilibrium strategy profiles that emerge from a noncooperative game of incomplete information.

We translate this theoretical framework into an econometric one, which we use as a basis to study identification. Identification of utility parameters obviously depends on the researcher's a priori knowledge of social structures. Without any such prior knowledge, identification fails. This is the first basic identification result we establish. Our second set of results considers the case most commonly assumed in the applied literature, where a researcher has full prior knowledge of the social structure. In a generalization of Bramoullé, Djebbari, and Fortin (2009) we show that in this case, when the researcher has access to individual data, identification of the structural parameters is generic in a sense we make precise. This casts the non-identification results that have been at the centre of much of the conventional econometrics literature in a new light, since they pertain to non-generic models which have no obvious theoretical rationale. With full prior knowledge of social structure but access to only aggregate data, first moments do not enable identification. However, building on approaches proposed by Glaeser, Sacerdote, and Scheinkman $(1996,2003)$ and formalized by Graham (2008), we show that second moments do.

The assumption of full prior knowledge of social structure, although routinely imposed in empirical work, may be conceptually untenable. Our third set of identification results explore how far one can get with partial prior knowledge of social structure. These results, motivated in part by the availability of social network 
data, indicate that when a priori information regarding the intensity of social ties between individuals is absent, prior knowledge of the mere existence (or absence) of ties between individuals enables identification. Identification for this case bears a conceptual resemblance to classical rank and order conditions for identification in linear simultaneous equations models (cf. Fisher (1966)), but the structure of the social interactions framework means that there are interesting differences from the standard results. Our results indicate that much more general models of social interactions can be employed in empirical work than has been done previously, when individual-level data are available. At the same time, we argue that there are limits to identification when data are comprised of individual observations and group level averages.

Finally, we address the issues of endogenous network formation, and the presence of public variables observable to those in the network but unobservable to the researcher. We treat endogenous network formation as the first stage of a two-stage game in which our general linear social interactions model describes payoffs from choices in the second stage. We show how the implications for identification of endogenous network formation entirely depend on the information available to agents at the time of network formation, so that for a number of interesting cases endogeneity does not matter. For the case where our results no longer apply, we indicate that the dealing with endogeneity involves the construction of the equivalent of the control function invented in Heckman (1979) and extended in Heckman and Robb (1986). This focus on information and network formations allows us to provide identification results for environments in which networks are durable in a sense we make precise. This places the endogeneity issue in the realm of a particular information asymmetry between the analyst and the population of agents

Two previous studies are relatively close to this one. (We discuss others in the context of our results later on.) Bramoullé, Djebbari, and Fortin (2009) consider identification for known social structures. We provide a mild generalization of their results by allowing for distinct social structures for contextual and endogenous effects, i.e. the effect of network members' exogenous characteristics and endogenous behaviors, respectively, on individual behavior. Further, they do not study identification when one does not know the complete social structure and when it is endogenous. Blume et al. (2011) anticipates some of our analysis. We employ a more general preference structure than that paper in that we allow for distinct social structures for different types of social interactions. Our results on identification under partial knowledge of the social structure are completely new as are our results on identification under aggregation and our discussion of endogeneity and information asymmetries between the analyst and the agents under study.

Throughout the paper, we will employ social interactions effects between students as an example in order to interpret assumptions and findings. The evidence for social interactions in education is well surveyed in Epple and Romano (2011) 
and Sacerdote (2011). This empirical literature is large, exploring social influences on educational and other outcomes. It includes a range of environments that fall into the general framework we study. For example, it is common to assume that individual outcomes are determined by unweighted averages of peer outcomes and/or characteristics, with definitions of peer groups ranging from self-identified friendships (Patacchini, Rainone, and Zenou 2012) to classmates (Graham 2008) to schoolmates (Bifulco, Fletcher, and Ross 2011) to Zip Codes (Corcoran et al. 1992).

In section 2 we develop a social interactions game of incomplete information whose Bayes-Nash equilibrium produces linear strategy profiles. Section 3 introduces additional assumptions that are needed to study these equilibrium strategy profiles as econometric models of individual outcomes. Section 4 studies identification based on complete knowledge of the social structure that connects agents in the population. Section 5 provides conditions under which identification will hold for partial knowledge of social structure. Section 6 considers the implications of alternate formulations of unobserved heterogeneity due to endogenous network formation and information differences between the information sets of agents and the analyst. Section 7 concludes.

\section{MicRofoundations}

In this section, we set up a theoretical model from which the econometric model we subsequently study is directly derived. We consider a Bayesian game - a social interactions game - in which the population of network members is a set $V$ containing $N<\infty$ members. Each individual $i$ is described by a vector of characteristics $\left(x_{i}, z_{i}\right)$, where $x_{i} \in \mathbf{R}$ is a publicly observed characteristic, and $z_{i} \in \mathbf{R}$ is a private characteristic observable only to individual $i{ }^{2}$ An individual's type $t_{i}$ is a vector $\left(x, z_{i}\right) \in R^{N+1}$, which details $i$ 's observable and unobservable characteristics, and the observable characteristics of everyone else. The vector of players' types is $(x, z) \in \mathcal{T}=\mathbf{R}^{\mathbf{2 N}}$. The a priori distribution of types is an exogenous probability distribution $\rho$ on $\mathcal{T}$. Knowledge of $\rho$ is common to all individuals, and each individual's beliefs about the types of others is a conditional distribution of $\rho$ given the individual's type.

Utility depends on an individual's own action and characteristics as well as network members' actions and characteristics. Individual $i$ chooses an action

\footnotetext{
${ }^{2}$ We restrict attention to one observable and one unobservable characteristic in order to simplify notation. All of our results are easily extended to the case when each of these is a vector.
} 
$\omega_{i} \in \mathbf{R}$ to maximize utility:

$$
\begin{aligned}
U_{i}\left(\omega_{i}, \omega_{-i}\right)=\left(\gamma x_{i}+z_{i}+\delta \sum_{j} c_{i j} x_{j}\right) \omega_{i}-\frac{1}{2} \omega_{i}^{2} & \\
& -\frac{\phi}{2}\left(\omega_{i}-\sum_{j} a_{i j} \omega_{j}\right)^{2} .
\end{aligned}
$$

Utility is separable into two components. The first line is the private component of utility and the second is the social component. Both are strictly concave in individual $i$ 's action. Marginal private utility is linear in individual $i$ 's own observable characteristic $x_{i}$ and private characteristic $z_{i}$. The term $\delta \sum_{j} c_{i j} x_{j}$ captures contextual effects - the direct influence of others' characteristics on $i$ 's choices. It is a weighted average of the characteristics of neighbors in a contextual-effects network. In our model, endogenous, or peer effects, come from social pressure, that is, social norms. This is described in the second line as the squared distance between individual $i$ 's behavior $\omega_{i}$ and the average $\sum_{j} a_{i j} \omega_{j}$ of the behaviors of his peers in an peer effects network. The parameter $\phi$ determines the marginal rate of substitution between the private and social components of utility.

The matrices $A$ and $C$ whose elements $a_{i j}$ and $c_{i j}$ determine peer and contextual effects are weighted adjacency matrices or weighted sociomatrices for the peer- and contextual-effects network, respectively. Each has dimension $N \times N$ and the magnitudes of the matrix elements measures the strength of network ties. The networks themselves can be described by graphs: the peer-effects network $\mathcal{A}$ has vertex set $V$ and edge set $E=\left\{(i, j): a_{i j}>0\right\}$. The contextual-effects network $\mathcal{C}$ is defined similarly with $C$ instead of $A$.

In the schooling example, the network is the population of $N$ students. Each student $i$ choses a level of effort. Observable characteristics include indicators of socio-economic status, such as family income. Contextual effects emerge because families with resources may contribute public goods such as school supplies or volunteer time to the classroom. The unobserved type $z_{i}$ may capture individual characteristics such as ability and family values concerning education, and can include common influences such as teacher quality. The peer effect is understood here to be a pressure to conform. Peer- and contextual-effects networks may differ, then, because the entire classroom may benefit from such things as parent volunteers, while peer effects come only from a student's friends.

Our utility function nests examples in the literature such as Davezies, d'Haultfoeuille, and Fougère (2009) and Manski and Mayshar (2003). Our model is closest to Blume et al. (2011) but strictly nests it because we allow for distinct peer-effect and contextual-effect sociomatrices and because we work with much weaker error restrictions than the i.i.d. assumption made by the earlier paper. This weakening is important in moving from the theoretical to the econometric model. 
Since all actions are chosen simultaneously, an equilibrium concept is required. We see this as an incomplete information game, and look for a Bayes-Nash equilibrium. That is, individuals choose an action to maximize the expectation of their utility given their type and the public types of others. The Bayesian game formalism assumes that the description of the game $\langle U, \gamma, \delta, \phi, A, C, \rho\rangle$ is common knowledge among individuals. Futhermore, we assume that $x$ is common knowledge, and each individual alone observes his private $z_{i}$. Equilibrium beliefs are constructed from the individuals' strategy functions and the common prior belief. The following axioms ensure the existence of a Bayes-Nash equilibrium:

T. 1. $\phi \geq 0$. $A$ and $C$ are non-negative, for each $i \in V, \sum_{j} a_{i j}$ is either 0 or 1 , and similarly for $C$. For all $i \in V, a_{i i}=0$.

T. 2. Second moments of $\rho$ exist.

Axiom T.1 has several parts. The restrictions $\phi \geq 0$ and non-negative $A$ together impose a preference for behavioral conformity. Hence, a student is more likely to exert effort if classmates also exert effort. The analogous restriction on $C$ means the effect of exogenous characteristics is proportionate to the strength of a tie. So the age of a student's friends may matter more than that of acquaintances and may be positive or negative, but the sign of the contextual effects will be the same as the sign of $\delta$. The restriction that the row sums of the peer and contextual effects be either 1 or 0 means that individuals in the network either care about these variables as weighted averages, or the individual is a social isolate - a "loner". The restriction $a_{i i}=0$ ensures that $i$ does not affect $i$ 's behavior via the sociomatrix. This is an obvious assumption given that this component of utility captures the effect of others' behaviors and not own behavior. Our model is not circular in that a student does not directly affect his performance via his own performance. Axiom T.2 is necessary to ensure that expected utility is well-defined for a large class of strategies.

Formally, a strategy for individual $i$ is a function $f_{i}: \mathbf{R}^{\mathbf{N}+\mathbf{1}} \rightarrow \mathbf{R}$ that assigns a choice $\omega_{i}$ to each of his possible types $\left(x, z_{i}\right)$. Denote by $\mathcal{F}$ the set of all strategy profiles $f(x, z)=\left(f_{1}\left(x, z_{1}, \ldots, f_{N}\left(x, z_{N}\right)\right)\right.$ such that for each $x$ and $i$, $f_{i}(x, \cdot): z_{i} \mapsto \mathbf{R}$ is in $L_{\rho}^{2}{ }^{3}$ A Bayes-Nash equilibrium of the game is a vector of strategy profiles $f(x, z)$ such that each $f_{i}$ maximizes $\mathrm{E}\left(U_{i}\left(\omega_{i}, \omega_{-i}\right) \mid x, z_{i}\right)$, where the expectation is taken with respect to the strategies $f_{-i}$ and the common prior $\rho$.

Theorem 1. If the Bayesian game satisfies axioms T.1 and T.2, it has a unique Bayes-Nash equilibrium. The equilibrium strategy profile can be written

$$
f(x, z)=\frac{1}{1+\phi}\left(I-\frac{\phi}{1+\phi} A\right)^{-1}(\gamma I+\delta C) x+\mu(x, z)+\frac{1}{1+\phi} z
$$

\footnotetext{
${ }^{3}$ This means that the squared integral of $f_{i}(x, \cdot)$ with respect to $z_{i}$ exists and is finite, ensuring that preferences over strategies for the Bayesian game are well-defined.
} 
where $\mu_{i}(x, z)$ depends only on $x$ and $z_{i}$. If $z$ is independent of $x$, then $\mu_{i}(x, z)$ depends only on $z_{i}$. If the elements of the $z$ are all pairwise independent, then $\mu_{i}(x, z)$ depends only on $x$. If both are true,

$$
\mu(x, z)=\frac{1}{1+\phi}\left(\left(I-\frac{\phi}{1+\phi} A\right)^{-1}-I\right) \mathrm{E}(z),
$$

a constant vector.

Theorem 1 provides sufficient conditions for the existence of a unique BayesNash equilibrium to the game, whose individual strategy profiles obey the linear structure commonly assumed in the empirical literature. The first term in (2) describes endogenous, contextual, and direct own-effects of public types $x$. This is the focus of empirical research. The third term expresses the direct effect on equilibrium behavior of individuals' private types $z$. The second term, $\mu(x, z)$, is the effect of higher-order beliefs - individuals' expectations of others' private types, their expectation of others' expectations of their beliefs, etc. In general, $i$ 's higher order beliefs may be a function of $\left(x, z_{i}\right)$, others' characteristics and $i$ 's private type. This may be important when network membership is endogenous and $x$ and the $z_{i}$ 's are correlated. We consider this case in section 6 . When $x$ and $z$ are independent, however, $\mu(x, z)=\mu(z)$, i.e. each individual's expectation of others' private types depends only on his own private type. Our econometric models will make this assumption (E.4 below), and we will sweep these higher-order beliefs into the unobserved term in the regression equation.

There are generally speaking, two kinds of endogenous social interactions models. We have chosen to model social interactions as coming through social norms. We model this as a conformity effect. Another source of social interactions comes through strategic complementarities in production. In the education literature papers such as Epple and Romano (1998) and Calvó-Armengol, Patacchini, and Zenou (2009) take this approach in modeling peer effects. ${ }^{4}$ The interpretation of the peer effects in such papers is that the marginal cost of educational achievement is affected by peer composition. Such an approach has the utility function

$$
U_{i}\left(\omega_{i}, \omega_{-i}\right)=\left(\gamma x_{i}+z_{i}+\delta \sum_{j} c_{i j} x_{j}\right) \omega_{i}+\phi \sum_{j} a_{i j} \omega_{i} \omega_{j}-\frac{1}{2} \omega_{i}^{2}
$$

Here the first two terms describe a production function that maps effort to an educational outcome. The second term is a strategic complementarity. The hard work of other students spills over to increase the marginal product of student $i$ 's effort. This is plausible for all kinds of reasons (see Sacerdote (2011)). The third term is the disutility of effort. The proof of theorem 1 applies to this model too, and an equilibrium exists and is unique for $0 \leq \phi<1 .^{5}$ The difference between

\footnotetext{
${ }^{4}$ Epple and Romano (2011, sec. 2.1) surveys different models of externalities between students.

${ }^{5}$ This model becomes more complicated when $\phi \geq 1$, and so this case is assumed away in the literature.
} 
this model and that of equation (1) is that here the vector $x$ enters the equilibrium strategy profile through the term $(I-\phi A)^{-1} x$, that is, $\phi$ rather than $\phi /(1+\phi)$ multiplies the matrix $A$. Thus the two models cannot be distinguished in the joint distribution of $x$ and $\omega$ without further assumptions. This renders moot the issue of identifying the source of endogenous social interaction. Hence we use the theoretical model outlined in (2) as our basis for econometric analyses of linear social interactions. We make this transition in the next section.

\section{From a Theoretical to an ECONOMETRIC MOdEL}

An econometric evaluation of our theoretical model requires additional assumptions. In this section we introduce these assumptions and discuss their role in identification of the utility parameters. Loosely speaking, parameters are "identified" if the map from utility parameters into the joint distribution of regressors and outcomes is one-to-one. The following definition, due to Koopmans (1953), is useful in translating our theoretical framework to an econometric one.

Definition 1. A structure $s$ for the linear social network model is a list $\langle\gamma, \delta, \phi, A, C, \rho\rangle$, where $\gamma, \delta$ and $\phi$ are utility parameters, $A$ and $C$ are peer- and contextual-effects sociomatrices, and $\rho$ is the a priori probability distribution on $\mathbf{R} \times \mathbf{R}$. A model is a set of structures.

The empiricist is interested in whether the utility parameters $\gamma, \delta$ and $\phi$ are identified in a model in which a number of sometimes implicit restrictions have been imposed on $A, C$ and $\rho$. Here, we make these restrictions explicit by concerning ourselves with the model $\mathcal{M}$, all of whose structures satisfy T.1-T.2 and the following assumptions:

E. 1. The support of the marginal distribution of $x$ has dimension $N$.

E. 2. For all $i$ and $j, a_{i j}>0$ iff $a_{j i}>0$. For some $i$ and $j, a_{i j}>0$.

E. 3. For all $i$ and $j, c_{i j}>0$ iff $c_{j i}>0$. For some $i$ and $j, i \neq j, c_{i j}>0$.

E. 4. For all $i, j \in V, x_{j}$ and $z_{i}$ are uncorrelated.

E. 5. At least one of $\gamma$ and $\delta$ is nonzero.

E. 1 ensures that the $N \times N$ matrix of parameters post-multiplied by $x$ in equation (2) is unique. Assumptions E.2 and E.3 place additional restrictions on the sociomatrices. While these are not necessary conditions in our theorems, they greatly simplify derivations. Each is, in itself, weak and only serves to eliminate knife-edge cases. The first parts of E.2 and E.3 require that when $j$ exerts social influence on $i, i$ also exerts social influence on $j$. These are weak assumptions because nonzero elements of the sociomatrices are allowed to be arbitrarily small and the strength of ties between two individuals may be asymmetric. We do not require these two assumptions for most of our results, including those which rely on holes in the network for identification. However, we prefer to maintain them because they greatly simplify proofs. The second parts of E.2 and E.3 require, 
respectively, that there exist at least one pair of agents who exert peer effects on each other and one pair of agents who exert contextual effects on each other. This rules out $C=I$. E.4 is a standard exogeneity condition with respect to $x$. In the context of our theory model, it means that higher order beliefs depend only on own-types, so $\mu(x, z) \equiv \mu(z)$. We will relax this assumption in section 6 . E.5 eliminates the special case in which the $x$ 's have no effect on the outcomes. Lemma 1 in the appendix shows that in this special case identification fails, so we ignore it in subsequent discussion.

For what follows, it will be convenient to define

$$
\begin{aligned}
\mu & =\mathrm{E}\left(\mu(z)+\frac{1}{1+\phi} z\right), \\
\varepsilon & =\mu(z)+\frac{1}{1+\phi} z-\mu, \\
B_{\phi}(s) & =\frac{1}{1+\phi}\left(I-\frac{\phi}{1+\phi} A\right)^{-1},
\end{aligned}
$$

and

$$
B(s)=B_{\phi}(s)(\gamma I+\delta C) .
$$

For structures in models satisfying assumptions T.1-T.2 and E.1-E.4, the equilibrium equation system of theorem 1 becomes

$$
\omega=\mu+B(s) x+\varepsilon,
$$

With this change of variables, the residual term $\varepsilon$ has unconditional mean 0 . Although it should not be forgotten that both $\mu$ and the distribution of $\varepsilon$ depend upon $\rho$, we shall not be using either for identification (except in section 4.2). Instead, we will identify parameters through the matrices $B(s)$. We index these matrices by $s$ to emphasize that it is from the structure that we will recover utility parameters.

Equation (5) may be contrasted with a purely statistical model of the form

$$
\omega=\alpha+B x+e,
$$

in which $e$ is constructed to be orthogonal to $(1, x)$. Viewing this statistical model through the prism of the game of section 2 and the econometric assumptions of this section has three advantages. First, it imposes some parameter restrictions on the model (e.g. the row sums of $B$ will be identical). Second, it facilitates the interpretation of parameter values in terms of commonly accepted models of interactive decision making. Third, it allows for causal conclusions from parameter estimates because it makes clear what environmental perturbations leave the structure unchanged.

From E. 1 it is immediate that $\alpha, B$ and $\operatorname{Var}(e)$, the covariance matrix of the reduced form errors from equation (6), summarize the relevant information for identification via the first and second moments of the data, and that each is unique. For 
purposes of identification, these moments are the objects that the data provide to the analyst.

Most of this paper is concerned with identification of the utility parameters of structures from the matrices $B(s)$ of equation (5), which describe how equilibrium strategy profiles vary with characteristics $x$.

Definition 2. Utility parameters $\gamma, \delta$ and $\phi$ are identified in a model $\mathcal{M}$ by $B$ if for all $s, s^{\prime} \in \mathcal{M}$, if $B(s)=B\left(s^{\prime}\right)$ then $(\gamma, \delta, \phi)=\left(\gamma^{\prime}, \delta^{\prime}, \phi^{\prime}\right)$.

Our identification definition ignores the constant term because, in comparing the equilibrium strategy profile (5) with the statistical model (6), without restrictions on $\rho_{\varepsilon \mid x}$ (the marginal prior of $\varepsilon$ given $x$ ), the individual-specific constant terms cannot provide additional information on $\gamma, \delta$ and $\phi$.

Since $B$ depends on the structure only through $\gamma, \delta, \phi, A$ and $C$, identification of the utility parameters will obviously depend on what is known about $A$ and $C$ a priori. Without a priori information about structures identification will fail since the inverse image of a matrix $B$ under the map $s \mapsto B(s)$ could contain structures with very different sociomatrices. In section 4.1 we assume that the pair $(A, C)$ is known a priori, while in section 5.1 , a priori knowledge only pertains to $C$. Let $\mathcal{M}(A, C)$ and $\mathcal{M}(C)$ denote the sets of all $s \in \mathcal{M}$ with fixed sociomatrices $A$ and $C$, and with fixed contextual-effects sociomatrix $C$, respectively. These should be thought of as sub-models of $\mathcal{M}$. For instance, when $A$ and $C$ are known a priori, the identification exercise is that of identifying the utility parameters in the set of structures $\mathcal{M}(A, C)$. It follows from these definitions that anything identified in $\mathcal{M}$ is identified in $\mathcal{M}(C)$ for every contextual-effects matrix sociomatrix $C$, and anything identified in $\mathcal{M}(C)$ is identified in $\mathcal{M}(A, C)$ for every peer-effects sociomatrix $A$. Let $M_{A}$ and $M_{C}$ denote the set of all peer- and contextual-effects sociomatrices, respectively, that satisfy the relevant parts of T.1-2 and E.1-5.

We first establish a basic identification result. This result supposes the following structure on observations:

\section{K. 1. For all $i$, the analyst observes $\left(\omega_{i}, x_{i}\right)$}

Without any a priori knowledge other than T.1-2, E.1-E.4 and K.1, the reduced form parameters $B=B(s)$ and the sum $\beta=\gamma+\delta$ are nonetheless identified; that is, they are identified in $\mathcal{M}$. This is critical. The remainder of the paper is concerned with the unpacking of $B=B(s)$ to recover utility and social interactions parameters. The theorem goes on to state that the parameter set $\gamma=\delta=0$ is identified in $\mathcal{M}$. A third result states that with an additional piece of a priori information, the set $\delta=\phi=0$ is identified, and in this case, by virtue of the first result, $\gamma$ is identified as well.

Theorem 2. Let $\mathcal{M}$ denote the model satisfying axioms T.1-T.2, E.1-E.4 and K.1. 
i. The matrix $B(s), \mu$, and the sum $\beta=\gamma+\delta$ are identified in $\mathcal{M}$ from the joint distribution of $\omega$ and $x$ without any additional a priori information.

ii. $\mathrm{E}(\omega \mid x)$ is independent of $x$ if and only if $\delta=\gamma=0$.

iii. If $C$ is known a priori and it is known a priori that there are two individuals $i$ and $j$ for whom $c_{i i} \neq c_{j j}$, then for all $i, \mathrm{E}\left(\omega_{i} \mid x\right)=\mathrm{E}\left(\omega_{i} \mid x_{i}\right)$ if and only if $\delta=\phi=0$. In this case, $\gamma$ is identified as well.

Otherwise, the parameters $\gamma, \delta, \phi, A, C$ are not identified without additional a priori information.

These results do not require E.5. But having dispensed with this case, it is convenient for expository purposes to maintain E.5 for the remainder of the paper. Note too that in case iii. it is not necessary to know the values of any elements of $C$, but merely that two diagonal values are distinct.

Theorem 2 is a negative result from the perspective of identifying social interactions. The non-identifiability of $\delta$ and $\phi$ means that the structural parameters that characterize the presence of the two possible social effects, peer and contextual, cannot be recovered given the assumptions we have made so far. To understand why, consider the following econometric specification, which is delivered from the first-order conditions for expected utility maximization in the Bayes-Nash equilibrium:

$$
\omega_{i}=\frac{\gamma}{1+\phi} x_{i}+\frac{\delta}{1+\phi} \sum_{j} c_{i j} x_{j}+\frac{\phi}{1+\phi} \sum_{j} a_{i j} \mathrm{E}\left(\omega_{j} \mid x\right)+\frac{1}{1+\phi} \varepsilon_{i}
$$

This system of $N$ equations is just a classic simultaneous equations system except that expectations of endogenous variables appear on the right hand side of the equation rather than realizations. The non-identification of this simultaneous equations system is a classical result - one that is unaffected by the row summability of $A$ and $C$. In fact one can interpret two stage least squares as making exactly this substitution. From this vantage point, identification failure stems from the absence of exclusion restrictions in the system. (See Bramoullé, Djebbari, and Fortin (2009) for elaboration of this intuition.)

We close this section by showing how a number of existing models constitute special cases of our general framework. The social interactions literature has focused on equation (7), the first order conditions for expected utility maximization, rather than the equilibrium strategy profiles. Hence our first two examples focus on econometric models that may be interpreted be interpreted as special cases of (7). Our third example illustrates how our models instantiates the idea of weak versus strong ties, a sociological distinction which is important for a variety of economic network analyses.

Example 1: linear-in-means models. In many social networks models, individuals are partitioned into non-overlapping groups $g$. Let $n^{g}$ denote the size of group $g$. In the linear-in-means model, an individual's behavior depends on his average 
group characteristics and average group behavior. This amounts to imposing the following constraints on the sociomatrices:

$$
\begin{aligned}
& c_{i j}=\frac{1}{n^{g}} \text { if } i, j \in g, \\
& a_{i j}=\frac{1}{n^{g}-1} \text { if } i, j \in g, \\
& c_{i j}=a_{i j}=0 \text { if } i \in g, j \notin g
\end{aligned}
$$

Combined with the assumption that $\mathrm{E}(\varepsilon \mid x)=0$, the first order conditions (7) may be rewritten:

$$
\begin{aligned}
\omega_{i}=\frac{\gamma}{1+\phi} x_{i}+\frac{\delta}{(1+\phi)\left(n^{g}-1\right)} & \sum_{j} x_{j} \\
& +\frac{\phi}{(1+\phi)\left(n^{g}-1\right)} \sum_{j \neq i} \mathrm{E}\left(\omega_{j} \mid x\right)+\frac{1}{1+\phi} \varepsilon_{i}
\end{aligned}
$$

Manski's (1993) study of identification of social effects is based upon a large sample approximation of this model, in which for all $i, n^{g} \rightarrow \infty$. In the limit

$$
\omega_{i}=\frac{\gamma}{1+\phi} x_{i}+\frac{\delta}{1+\phi} \bar{x}^{g}+\frac{\phi}{1+\phi} \mathrm{E}\left(\bar{\omega}^{g} \mid x\right)+\frac{1}{1+\phi} \varepsilon_{i}
$$

where $\bar{x}^{g}$ and $\bar{\omega}^{g}$ are group-level averages of the respective variables.

The unweighted averaging assumed in the linear-in-means model does not have a theoretical justification but rather reflects a modeling choice made for simplicity, or because of limits on what is observable about the groups. It is trivial to think of contexts in which weights will not be equal. For high school students, one could easily imagine differences in sociomatrix elements that reflect relative popularity, strong versus weak friendships, and the like. One message of this paper will be that it is not necessary to rely on the simplification of unweighted averaging. While some prior information on the sociomatrices $A$ and $C$ is necessary for identification of the utility parameters, the necessary information is less than that assumed in the linear-in-means model.

Our framework can also be used to assess the interpretability of different variations of (7) with respect to rigorous microfoundations. For example, a major empirical study of educational peer effects is Sacerdote (2001), which examines roommate pair interactions at Dartmouth. Sacerdote assumes that each student $i$ 's grade point average depends on his own ability and the ability and grade point average of his roommate $j$. Sacerdote is careful to allow for measurement error in ability. We ignore this for simplicity, since in its absence his model reduces to

$$
\omega_{i}=d_{0}+d_{1} x_{i}+d_{2} x_{j}+d_{3} \omega_{j}+s_{i} \text {. }
$$

Sacerdote follows the theoretically appropriate formulation of endogenous social effects by employing the average of each individual's roommates, which for pairs is simply the outcome of the other roommate. Further, given that there is only a 
single roommate, there is no issue of the restriction of the linear-in-means model $A$ matrix. On the other hand, as in Lee (2007), the inclusion of $\omega_{j}$ rather than $\mathrm{E}\left(\omega_{j}\right)$ begs the question of what information sets are available to agents, since a roommate's grades are not observable contemporaneously. That said, there is a simple reinterpretation of this model as

$$
\omega_{i}=d_{0}+d_{1} x_{i}+d_{2} x_{j}+d_{3} \mathrm{E}\left(\omega_{j}\right)+d_{3}\left(\omega_{j}-\mathrm{E}\left(\omega_{j}\right)\right)+s_{i}
$$

which is isomorphic to our equilibrium best response function when roommates are playing a Bayes-Nash game. Does this do violence to Sacerdote's analysis? We argue that it does not, since instrumenting for $\omega_{j}$ is equivalent to replacing this variable with $\mathrm{E}\left(\omega_{j}\right)$. Note that in this specification, $d_{2}$ and $d_{3}$ are not identified if $\mathrm{E}\left(\omega_{j}\right)$ is determined by a linear combination of $x_{i}, x_{j}$ and a constant. (See corollary 1 for a further discussion.) Identification of $d_{2}+d_{3}$ holds and is a special case of theorem $2{ }^{6}$

Example 2: linear-in-means models based on neighborhoods. A second common approach to analyzing social effects has extended the linear- in-means model by exploiting observed network data to locate individuals in neighborhoods, and using these neighborhoods to generate sociomatrices. One example of this strategy is De Giorgi, Pellizari, and Redaelli (2010) who employ administrative data from university students to explore peer effects among classmates, where interactions are determined by overlapping classroom enrollments. A similar approach is employed in Calvó-Armengol, Patacchini, and Zenou (2009), using AddHealth data.

Formally, let $i$ 's neighborhood $h$ be the set of other agents to whom he is connected and let $n^{h}$ be the number of agents in this set. Note that $i \notin h$. The weights associated with a linear-in-means model based on neighborhoods correspond to

$$
\begin{aligned}
& c_{i j}=\frac{1}{n^{h}} \text { if } j \in h, \\
& a_{i j}=\frac{1}{n^{h}} \text { if } j \in h, \\
& c_{i j}=a_{i j}=0 \text { if } j \notin h
\end{aligned}
$$

The reduced form regression that is generated by the addition of these assumptions to our framework is

$$
\omega_{i}=d_{0}+d_{1} x_{i}+d_{2} \bar{x}^{h}+d_{3} \mathrm{E}\left(\bar{\omega}^{h}\right)+\varsigma_{i}
$$

where $\bar{x}^{h}$ and $\bar{\omega}^{h}$ denote averages for neighborhood $h$. While equation (12) may resemble equation (7), it in fact implies a much richer structure for social interactions. Unlike the linear-in-means model, agents are no longer partitioned into

\footnotetext{
${ }^{6}$ Sacerdote (2001) shows that identification can hold under restrictions on the unobservables in his model. In our formulation of his model, this would require that $\omega_{j}-\mathrm{E}\left(\omega_{j}\right)$ and $\zeta_{i}$ are uncorrelated, which Sacerdote (2001) notes involves the very stringent and arguably non-credible requirement that $\zeta_{i}$ and $\zeta_{j}$ be uncorrelated.
} 
non-overlapping groups; an agent to whom many are connected has a larger influence than one to whom few are connected because of differences in the number of neighborhoods the respective agents inhabit.

However, the formulation is still restrictive relative to our general $C$ and $A$ sociomatrix formulation as (12)'s generalization of (7) involves the relaxation of the block diagonality assumption of the linear-in-means model but retains equal values of the non-zero elements of each row of the implied sociomatrix. This could be an inaccurate, not to mention excessively restrictive, representation of social interactions.

In this example too, our microfoundations can be used to evaluate the statistical formulations of (12). For example, De Giorgi, Pellizari, and Redaelli (2010) use $\bar{\omega}^{h}$ rather than $\mathrm{E}\left(\bar{\omega}^{h}\right)$. As argued above, our Bayes-Nash formulation is more natural and does no violence if employed to interpret their regression. Further, the assumption $c_{i i}=0$ may not be natural.

Calvó-Armengol, Patacchini, and Zenou (2009) make a more substantial deviation from our framework. They develop a complete-information social interaction game where individuals respond to the choices of peers in their social network. Individuals utilities are additively separable in two choice variables, private effort and peer-induced effort. These are perfect substitutes in the production of observed output (which is not an argument of the utility function). Their model differs from ours in two important way. First, they assume that individual characteristics (including contextual effects) affect only the utility of private effort, and so they elide the identification problem since individual characteristics do not feed back into peer effects. So, for instance, if a student in a classroom had an exogenous improvement in health status that reduced his cost of effort, and so he chose to work more, this would have no effect on others' effort levels or outcomes. Second, although individual characteristics are not an argument of peer effort utility, there is heterogeneity in equilibrium peer effort nonetheless, because ceteris paribus the marginal utility of peer effort is assumed to scale linearly with the number of connections one has - individuals with more contacts are assumed to be more susceptible to peer pressure. These unusual modeling choices serve a purpose. Calvó-Armengol, Patacchini, and Zenou make a direct connection between the peer-effort choice and sociological measures of centrality. They derive that the equilibrium peer effect equals the Katz-Bonacich centrality vector.

This and other measures can be derived from our $A$ matrix, and both models have the advantage of deriving the rate of attenuation of influence that is a key parameter of these measures from the marginal rate of substitution between private and social components of utility.

Example 3: strong and weak ties. Empirical work by economists on networks has largely concerned networks with only one type of connection between agents. Sociologists, on the other hand, have recognized that social connections may 
have different manifestations, and that the distribution of different kinds of connections in a social network has an impact on network outcomes. Perhaps the most well-known distinction among connections is that of strong and weak social ties. Granovetter (1973) argued that weak ties play an important role in job search because they relay useful job information more frequently. Lin (2002) suggests that weak ties are useful because weak-tie job referrals are drawn from a different and often better distribution of openings. Montgomery (1994) has embedded simple two-edge-type social networks into job search models to investigate the impact of the distribution of weak versus strong ties on employment rates and wage distributions. While the labor-market literature extolls the virtues of weak ties, in other aspects of economic life strong ties may be more important. Some ethnographic work suggests that strong ties have more value to poor individuals than weak ties. The suggestion is that the poor, lacking access to markets, rely more on reciprocity in their social networks for the provision of credit and a variety of commodity flows (see Granovetter (1973, pp. 209-13).)

The flexibility of weighted sociomatrices allows for the empirical distinction between strong and weak ties. Suppose that an individual $i$ has $n_{i s}$ strong ties and $n_{i W}$ weak ties. Suppose too that the ratio of the strength of strong to weak ties is $\theta$. Define elements of the peer- effects sociomatrix as

$$
a_{i j}= \begin{cases}1 /\left(n_{i W}+\theta n_{i S}\right) & \text { if } j \text { is weakly tied to } i \\ \theta /\left(n_{i W}+\theta n_{i S}\right) & \text { if } j \text { is strongly tied to } i \\ 0 & \text { otherwise. }\end{cases}
$$

A statistical model with this kind of network structure can be estimated from survey data that includes information on tie strength or data on ties that would allow a researcher to infer the nature of the tie. The AddHealth data set is an obvious candidate for constructing weak versus strong ties. Patacchini, Rainone, and Zenou (2012) in fact explore this possibility by assuming that the weights on friends report in the data are linearly declining in the order listed by each student and by studying the differences between friendships report in two survey waves versus one.

\section{IDENTIFICATION WITH KNOWN SOCIOMATRICES}

In this section, we describe identification of the primitive utility parameters $\gamma, \delta$ and $\phi$ when the sociomatrices $A$ and $C$ are known to the analyst. We do not take a stance on the source of this a priori knowledge. It may be the case that the matrices are empirical constructions or chosen for theoretical reasons. Formally, we augment the assumptions made in sections 2 and 3 with

K. 2. $A$ and $C$ are exogenous and known to the analyst a priori. 
K.1 defines the information assumption on the sociomatrices maintained in this section. The assumption that the analyst knows the values of the sociomatrices is a strong one, and we believe that standard approaches to generating a priori values of $A$ and $C$ are often theoretically unjustified. However, since this is in fact how the bulk of the social interactions and networks literatures has proceeded, it is important to understand identification for such contexts. K.2 says that the analyst observes both the outcomes and characteristics of each member of the population. We will relax this assumption when we consider identification with aggregated data in Section 4.2.

One major result of this section is that when individual data are available, there is a precise sense in which identification of the primitive utility parameters of the linear social networks models is "typically" the case. In this section we say that "parameters are identified for generic pairs of sociomatrices" if the set of sociomatrix pairs $(A, C)$ for which utility parameters are not identified is a lowerdimensional subset of $M_{A} \times M_{C}$. We show this below when $A$ and $C$ are known a priori. Our results indicate that concerns over simultaneity as a source of nonidentification of social effects are misplaced when $A$ and $C$ are known, unless one has a justification for working with a model from the non-generic (small) set of linear social network models in which identification fails.

We further consider identification when data are aggregated. Consideration of this case was initiated by Glaeser, Sacerdote, and Scheinkman (2003) and formalized in Graham (2008). They employ versions of the linear-in-means model and focus on identifying the equivalent of the contextual-effects parameter $\delta$ under the assumption that $\phi=0$, i.e. peer effects are not present. Here we provide identification results that generalize the cases these authors studied. We show that identification is possible when both effects are present and for social structures other than the linear-in-means specification.

4.1. Individual-level data. We begin by generalizing an important result due to Bramoullé, Djebbari, and Fortin (2009), which places conditions on the sociomatrices that are sufficient for identification of the parameters. Our generalization accounts for distinct peer- and contextual-effects sociomatrices. The result provides conditions such that the matrix $B$ in the statistical model (6), when identified with $B(s)$ in the structural equation (5), can be used to back out the values of $\gamma, \delta$ and $\phi$. Since $A$ and $C$ are known a priori, "is identified" means in this subsection "is identified in $\mathcal{M}(A, C)$ ". The conditions, however, are abstract, and so we elaborate on them. We show next that the set of $(A, C)$ pairs for which these conditions fail is a lower-dimensional subset of $M_{A} \times M_{C}$. Thus identification is the "typical case". We provide conditions on the structure of the underlying networks that are sufficient to guarantee identification of the utility parameters. These results can be seen as demonstrating how exclusion restrictions are sufficient for identification.

Theorem 3. Suppose T.1-T.2, E.1-E.5, and K.1-K.2. 
i. If $A, C$ and $A C$ are distinct, then linear independence of the matrices $I$, $A, C$ and $A C$ is necessary and sufficient for identification of $\gamma, \delta$ and $\phi$. The parameter $\gamma$ is identified if and only if $\delta$ is identified. ${ }^{7}$

ii. If $A \neq C$ and $A C=C$, then linear independence of $I, A$ and $C$ is necessary for identification of $\gamma, \delta$ and $\phi$, and sufficient to identify $\gamma$ and $\delta$. If $\gamma \neq 0$, then independence is also sufficient to identify $\phi$.

Case $i$ of theorem 3 says that the failure of identification implies the existence of a non-zero solution in $\lambda_{1}, \lambda_{2}, \lambda_{3}$ and $\lambda_{4}$ of the following equation system:

$$
\begin{aligned}
\lambda_{1}+\lambda_{2} c_{i j}+\lambda_{3} \sum_{j} a_{i j} c_{j i} & =0 \text { for all } i, \\
\lambda_{2} c_{i j}+\lambda_{4} a_{i j}+\lambda_{3} \sum_{j} a_{i j} c_{j i} & =0 \text { for all } i \neq j
\end{aligned}
$$

These linear dependence conditions implicitly define the set of matrices $A$ and $C$ such that identification fails. Our interest in theorem 3 lies in understanding whether sociomatrices $A$ and $C$ "almost always", "sometimes", or "almost never" satisfy these restrictions. Genericity of identification captures the idea of "almost always". ${ }^{8}$ The following definition explains what we mean when we say that utility parameters are generically identified.

Definition 3. The utility parameters $\phi, \gamma$ and $\phi$ are identified in a generic model with a priori known $A$ and $C$ if there is a closed subset $K$ of $M_{A} \times M_{C}$ of dimension less than that of $M_{A} \times M_{C}$ such that for all $(A, C) \in\left(M_{A} \times M_{C}\right)$ that are not in $K$, utility parameters $\gamma, \delta$ and $\phi$ are identified in $\mathcal{M}(A, C){ }^{9}$

It will be useful to know that the dimensions of the set of allowable $A$ and $C$ sociomatrices are $\operatorname{dim} M_{A}=N(N-2)$ and $\operatorname{dim} M_{C}=N(N-1)$, respectively. ${ }^{10}$ Identification in a generic model means that even when identification in $\mathcal{M}$ is impossible, we can identify utility parameters if we restrict the set of structures by discarding those with sociomatrices in a lower-dimensional subset of the allowed sociomatrices. The next result says that this is indeed the case when $A$ and $C$ are known a priori.

\footnotetext{
${ }^{7}$ Additional conditions on $A$ and $C$ imply that if any one parameter is identified, so are the other two. These conditions hold generically in $C$ for each $A$ and vice versa.

${ }^{8}$ Our results may be seen as a complement to McManus (1992) who established generic identification for parametric nonlinear models. He studies a space of nonlinear functions each of which is indexed by a parameter vector and employs a slightly different notion of genericity.

${ }^{9}$ The dimensions of sets in this definition are well-defined because these are semi-algebraic sets. Each is thus the finite union of manifolds, and the dimension is defined to be the largest dimension of these manifolds.

${ }^{10}$ Each row of $C$ has one restriction, that the sum be 1 or 0 , while each row of $A$ has two: that the sum be 1 or 0 , and that every diagonal element of the matrix equal 0 .
} 
Corollary 1. For each peer-effects sociomatrix $A$ such that the network contains a component ${ }^{11}$ of size at least 3 , there is generic subset of contextual-effects sociomatrices $C$ such that $\gamma, \delta$ and $\phi$ are identified in $\mathcal{M}(A, C)$.

This corollary states that utility parameters are identified in a generic model. To place this corollary in the education context, suppose that social interactions are confined to students in a given classroom. Interpret nonzero entries in the peer-effects sociomatrix $A$ as indicating direct friendship ties between students. Corollary 1 says that if there are three or more students in this classroom who are friends or friends-of-friends, for a generic contextual-effects sociomatrix $C$ the utility parameters are identified.

The proof of the corollary indicates that there are two ways that a pair $(A, C)$ of sociomatrices can cause a failure of identification. First, every individual is either isolated in the peer-effects network or is influenced by only one person. Second, for each $A$ there is a low-dimensional set of contextual-effects matrices $C$ for which identification can fail. This second collection of pairs has the larger dimension, but the dimension of this set is no more than $\operatorname{dim} M_{A}\left(\operatorname{dim} M_{C}-\right.$ 1 ). This is less than $\operatorname{dim} M_{A} \operatorname{dim} M_{C}$, the dimension of the set of all allowable sociomatrix pairs.

Corollary 1 , while striking, follows from the natural intuition that a priori knowledge of $A$ and $C$ radically decreases the number of unknown parameters in the best response function in equation (2). With $N$ equations describing individual behavior, we have $N$ exogenous variables. One would intuitively expect that the triple $(\gamma, \delta, \phi)$ be identified for $N \geq 3$. Identification fails when the sociomatrices are so symmetric that distinct relationships within the network become redundant. As lemma 3 in the appendix shows, this happens most prominently when $C=A$ and $A^{2}=A$.

Our finding that identification is generic for known sociomatrices contrasts with much of the conventional wisdom in the econometric literature on the identification of social effects. In particular, since Manski (1993), there has been a recognition that for certain models in which individual choices are a linear function of the averages of individual characteristics, identification can fail. Manski's (1993) demonstration that the utility parameters $(\gamma, \delta, \phi)$ are not identified for the large sample approximation (10) immediately follows from the fact that $\mathrm{E}\left(\bar{\omega}^{g} \mid x\right)$ is linearly dependent on 1 and $\bar{x}^{g}$. Manski dubbed this identification failure "the reflection problem", and it has dominated econometric work on social effects ever since. Note that as the size $n^{g}$ of each group $g$ becomes large, $\left|\left(A^{2}\right)_{i j}-A_{i j}\right| \rightarrow 0$ and $\left|A_{i j}-C_{i j}\right| \rightarrow 0$. The large sample approximation imposes the extreme symmetry condition described above, resulting in non-identification.

\footnotetext{
${ }^{11}$ A component is a maximal connected sub-network.
} 
Corollary 2 gives a sufficient condition that rests on information regarding the network rather than the specific values of the weights. Formally, this kind of information can be represented by a graph for each network with vertex set $V$ and a set of edges corresponding to non-zero elements in the relevant weighted sociomatrix. For instance, there is an edge from $i$ to $j$ in the peer-effects network $\mathcal{A}$ if and only if $a_{i j}>0$, and similarly for the contextual-effects network $\mathcal{C}$. Axioms E.2 and E.3 mean that we can represent these networks by undirected graphs. We write $i \sim_{A} j$ if there is an edge between $i$ and $j$ in the peer-effects network, $i \sim_{C} j$ if the edge exists in the contextual-effects network.

Corollary 2. Suppose there are distinct individuals $i$ and $j$ who are connected by a sequence of edges, some in the peer-effects network and some in the contextualeffects network, and who are not connected by a path in either the peer-effects network or the contextual-effects network alone. Then $\gamma, \delta$ and $\phi$ are identified.

This corollary demonstrates how restrictions on merely the existence of social interactions imply identification. Its conditions are satisfied whenever components of the networks overlap. ${ }^{12}$ For instance, if families attending a given school deliver contextual effects at the school level (e.g. they provide public goods) but peer effects do not cross classrooms or grade levels, then the utility parameters are identified. This condition cannot arise when one assume that $A=C$, and illustrates how the existence of richer peer and contextual social structures can facilitate rather than hamper identification.

Taken as whole, theorem 3 and corollaries 1 and 2 show, in our judgment, that concerns about non-identification with a priori knowledge of $A$ and $C$ are misplaced. Of course, our genericity finding in corollary 1 does not mean that a given model is identified. Rather, it says that if the utility parameters are not identified, then the researcher's choice of $A$ and $C$ is a very special case relative to the set of matrices that are consistent with the behavioral model we have described. It is always possible that a researcher has a principled reason for choosing sociomatrices under which identification fails. Our message is simply that such a reason needs to be present to conclude that the presence of social effects in preferences cannot be uncovered by the data.

Corollaries 1 and 2 are instances of case $i$. of theorem 3, and comparing them to previous work on identification and the linear-in-means model highlights the fragility of the reflection problem. Previous work has already produced variations of the linear-in-means model in which, unlike Manski's formulation, identification holds. Lee (2007), Lee, Liu, and Lin (2010), Bramoullé, Djebbari, and Fortin (2009) and Davezies, d'Haultfoeuille, and Fougère (2009) provide positive identification results based on (9), the exact linear-in-means model as opposed to the large sample approximation. Bramoullé, Djebbari, and Fortin (2009) and

\footnotetext{
${ }^{12}$ The exception is when the components of the peer-effects network are unions of components of the contextual-effects network.
} 
Davezies, d'Haultfoeuille, and Fougère (2009) study a version of Lee (2007), and find that if there are at least 3 groups of different size, identification holds for the exact model. Further, Bramoullé, Djebbari, and Fortin (2009) and Blume et al. (2011) show that the Manski non-identification result will hold if, contrary to our theoretical reasoning, $a_{i i}$ is non-zero, which in the linear-in-means case implies that if each agent reacts to an unweighted average of the expected choice, the reflection problem reemerges even if groups are finite - a conclusion that was anticipated in Moffitt (2001).

The following corollary is a mild generalization of previous results and is an instance of case ii of theorem 3.

Corollary 3. Suppose there is a component of the contextual-effects sociomatrix $C$ such that all $c_{i j}$ are equal and assume that any component of the peer-effects network is either a subset of or disjoint from the contextual-effects component. Suppose also that there exist two pairs of individuals $i \neq j$ and $k \neq l$ in the contextual-effects component such that $a_{i j} \neq a_{k l}$. Then $\gamma, \delta$ and $\phi$ are identified.

This corollary says that if there is a subset of the network such that the linearin-means assumption applies with respect to contextual effects and components associated with peer effects are either subsets or disjoint from this component, then identification will hold if two distinct pairs of individuals have different peereffects sociomatrix values. This illustrates how fragile the reflection problem is. In a classroom setting, for instance, if one takes the peer- and contextual-effects components to be the entire classroom as the linear-in-means model does, then a single inequality in $A$ involving non-overlapping pairs of students is sufficient for identification.

4.2. Aggregate Data. Individual-level data on social networks are often unavailable, or are incomplete due to sampling gaps. However, aggregate statistics are widely available (e.g.: average standardized test scores at the school-level, citylevel crime incidence, county-level unemployment rates, etc.). One approach that takes advantage of such data, originating with Glaeser, Sacerdote, and Scheinkman $(1996,2003)$ and later extended by Graham (2008), focuses on the informational content of cross-sectional data on group level averages.

To see how such data can be related to the linear social interactions model we have developed, consider data drawn from $G+1$ non-overlapping groups numbered $g=0, \ldots, G$. Each group $g$ contains $n^{g}$ members. We assume that the primitive utility parameters $\gamma, \delta$ and $\phi$ are constant across the groups in order to render the use of aggregate data interpretable, but that sociomatrices are groupspecific, so each group $g$ is associated with a distinct set of sociomatrices $A^{g}$ and $C^{g}$. For many contexts, heterogeneity in social structure seems natural across groups, even when populations are of the same size. One example is school classrooms, where one would naturally expect different social structures, even for classrooms of a given size. 
Denote by $\omega_{i}^{g}, x_{i}^{g}$ and $\varepsilon_{i}^{g}$ the outcomes, observed characteristics, and unobserved characteristics, respectively, of individual $i$ in group $g$ and let $\bar{\omega}^{g}, \bar{x}^{g}$ and $\bar{\varepsilon}^{g}$ be the group-level averages of these variables. The model is such that assumptions T.1-T.2, E.1-E.5 hold at the group level. In addition, we assume:

K. $\mathbf{1}^{\prime}$. For all $g, A^{g}$ and $C^{g}$ are exogenous and known to the analyst a priori.

K. $\mathbf{2}^{\prime}$. For all $g$, the analyst observes $\left(\bar{\omega}^{g}, \bar{x}^{g}\right)$.

Axioms $\mathrm{K} .1^{\prime}$ establishes what, in this section, is assumed to be the analyst's a priori knowledge. Axiom K.2' modifies K.2 so that the analyst only observes group-level averages of $x$ and $\omega$. For each group, let $\left(\omega^{g}, x^{g}, \varepsilon^{g}\right)$ denote the vectors of outcomes and observed and unobserved group characteristics for group $g$, respectively, and let $\tilde{n}^{g}$ denote the vector each of whose elements is $1 / n^{g}$. Given equations (5) and (4), $\bar{\omega}^{g}, \bar{x}^{g}$, and $\bar{\varepsilon}^{g}$ are related to the individual-level variables by

$$
\bar{\omega}^{g} \equiv \tilde{n}^{g} \omega^{g}=\tilde{n}^{g} \mu^{\varepsilon}+\tilde{n}^{g} B_{\phi}^{g}\left(\gamma x^{g}+\delta C^{g} x^{g}\right)+\frac{1}{1+\phi} \tilde{n}^{g} \mathcal{E}^{g}
$$

A natural starting point for many empiricists would be to estimate a linear-inmeans model, which amounts to imposing the restriction on (14) that the rows and columns of $A^{g}$ and $C^{g}$ each sum to 1 . This yields

$$
\bar{\omega}^{g}=\mu^{\varepsilon}+(\gamma+\delta) \bar{x}^{g}+\frac{1}{1+\phi} \bar{\varepsilon}^{g}
$$

It is easy to see from this equation that separate identification of the structural parameters from the joint distribution of $\bar{\omega}^{g}$ and $\bar{x}^{g}$ is not possible: $\delta$ and $\gamma$ enter the joint distribution only through the sum, and $\phi$ cannot be untangled from the variance of $\bar{\omega}$ under our current assumptions.

While the first moments do not permit identification, the key insight of Glaeser, Sacerdote, and Scheinkman and Graham is that, under further assumptions, second moments may. Glaeser, Sacerdote, and Scheinkman pointed out that conditional on $\bar{x}^{g}$, variation in $\bar{\omega}^{g}$ is consistent with the variation that would be predicted in averaging i.i.d. random variables. Their argument, which is heuristic, is that $\operatorname{Var}\left(\bar{\omega}^{g} \mid \bar{x}^{g}\right)$ will reveal social interactions by comparing the sample variances for different group sizes to one in which $\omega_{i}-\mathrm{E}\left(\omega_{i} \mid \bar{x}^{g}\right)$ is i.i.d. within and across groups.

Following Glaeser, Sacerdote, and Scheinkman (1996) and the case in Graham (2008) where group level effects are absent, we add the following additional constraint on the model in this section. ${ }^{13}$

E. 6. For all $i, j x_{i}^{g}$ and $x_{j}^{g}$ are i.i.d. and $\varepsilon_{i}^{g}$ and $\varepsilon_{j}^{g}$ are i.i.d.

\footnotetext{
${ }^{13}$ Graham (2008) has additional identification results in the case where random group effects are present.
} 
This is a stronger i.i.d. assumption than we have had so far, namely that for all $i, j, x_{i}^{g}$ and $\varepsilon_{i}^{g}$ are i.i.d. Together with E.6, equation (14) yields: ${ }^{14}$

$$
\operatorname{Var}\left(\bar{\omega}^{g}\right)=\frac{1}{\left(n^{g}\right)^{2}} \sum_{j \in g}\left(\sum_{i \in g} B_{i j}^{g}\right)^{2} \sigma_{x}^{2}+\left(\frac{1}{1+\phi}\right)^{2} \frac{1}{n^{g}} \sigma_{\varepsilon}^{2}
$$

Setting one group, $g=0$, as the baseline, define the following statistic for the remaining groups $g=1, \ldots, G$, where $\operatorname{Var}(x)=\sigma_{x}^{2}$ is observed

$$
v^{g}=\frac{n^{g} \operatorname{Var}\left(\bar{\omega}^{g}\right)}{\sigma_{x}^{2}}-\frac{n^{0} \operatorname{Var}\left(\bar{\omega}^{0}\right)}{\sigma_{x}^{2}}
$$

The following theorem shows that $v^{g}$ can be used for identification. Define $M_{A}^{g}$ and $M_{C}^{g}$ to be the set of peer- and contextual-effects sociomatrices for group $g$; that is, they satisfy the matrix assumptions T.1, E.2 and E.3.

Theorem 4. Assume T.1-T.2, E.1-E.6 and K.1'-K.2'. Suppose $\delta, \phi$, and $\beta=$ $(\gamma+\delta) \neq 0$. Suppose there are groups $0, \ldots, G$, that $G \geq 5$, and that for each group $g=1, \ldots 5, n^{g} \geq 3$ and $A^{g}$ is not bistochastic. Then the set $J$ of matrices $\left(C^{1}, \ldots, C^{5}\right)$ such that $v^{1}, \ldots, v^{5}$ does not identify the utility parameters $\gamma, \delta$ and $\phi$ is a closed lower-dimensional subset of $\prod_{g=1}^{5} M_{C}^{g}$.

Theorem 4 says when data are in the form of group averages, second moments can be used to identify the utility parameters provided that the $\left(A^{g}, C^{g}\right)$ pairs fulfill a condition on $C^{g}$ that, given $A^{g}$, holds generically. In addition, one needs a certain degree of variation across the sociomatrices to allow for the different groups to provide distinct second moments from which the utility parameters can be backed out.

The theorem builds on Graham (2008), who explores the case in which peer effects are effectively absent (for all $g, A^{g}=0$ ), and contextual effects are characterized by a linear-in-means structure where for all $g, c_{i i}=0$ (all of which are allowed by T.1-T.2 and E.1-E.5). The linear-in-means assumption reduces the number of required groups with distinct $C^{g}$ 's to 3 relative to the 5 in theorem 4 . Our result indicates that the logic of Graham's analysis extends beyond the linearin-means model. It does not require the absence of a peer effect. Neither does it require different group sizes, which in his analysis generates the necessary variation for identification. However, in our formulation, this comes at a cost: data loss in moving from individual to group-average observations necessitates a priori information on characteristic covariances. Although we conjecture that similar

\footnotetext{
${ }^{14}$ Equation (16) follows directly from

$$
\bar{\omega}^{g}=\mu^{\varepsilon}+\frac{1}{n^{g}} \sum_{j \in g}\left(\sum_{i \in g} B_{i j}^{g}\right) x_{j}^{g}+\frac{1}{1+\phi} \frac{1}{n^{g}} \sum_{i \in g}\left(\varepsilon_{i}^{g}-\mu^{\varepsilon}\right) .
$$
}


statistics can be constructed with more complicated covariance structures, here, we have assumed independence.

4.3. Mixed individual and aggregate data. We conclude this section by considering linear social interactions models that are based on a combination of individual-level and aggregate data. A number of studies, including many in the important first generation of empirical social interactions research, combine individual-data from the Panel Study of Income Dynamics (PSID) with averages of individual outcomes measured at the Zip Code level (Datcher 1982, Corcoran et al. 1992) or the census-tract level (Sharkey and Elwert 2011). This combination exploits the geographic identifiers in the PSID to construct measures that are interpreted as social influences. The sampling scheme for the PSID, when combined with aggregate information, produces regressions of the form

$$
\omega_{i}=b_{0}+b_{1} x_{i}+b_{2} \bar{x}^{g}+\eta_{i}
$$

where $g$ denotes the relevant level of aggregation. This regression, to be interpretable as an equilibrium strategy profile, implies assumptions of the form (8). Since the sampling scheme we describe provides no information on $A^{g}$ and $C^{g}$, this equation represents an information reduction relative to the row describing $\omega_{i}$ in equation (6), which we have already showed is not identified when these matrices are unknown. Relative to the $\omega_{i}$ row found in equation (6), equation (17) represents a misspecified regression so the parameters in (17) will depend on the underlying parameters $\gamma, \delta, \phi, A^{g}$ and $C^{g}$. The one positive use of (17) is that if $b_{2}=0$, then neither peer nor contextual effects are present in the preferences of agents.

\section{IDENTIFICATION WITH PARTIAL INFORMATION ON SOCIOMATRICES}

Theorem 2 states that without prior knowledge on the sociomatrices beyond what is necessary for the existence of a Bayes-Nash equilibrium in the quadraticpayoff game, there is little that can be learned about the preference parameters which constitute the primitives of the behavioral model. Section 4 explored the polar opposite case that is employed in most empirical applications, namely identification when these matrices are (assumed to be) known. We now explore the degree to which parameters can be identified with only partial knowledge of social interactions.

There are many ways in which one can model partial knowledge of $A$ and $C$. Two forms of partial knowledge are, in our view, particularly salient. First, the analyst may have a priori knowledge about $C$ without any a priori knowledge about $A$ beyond T.1 and E.2. This is a natural case to consider because peer effects embodied in $A$ represent a primitive psychological proclivity to behave similarly to others, for which theory provides no guidance. Such guidance may, however, exist for $C$. Classrooms provide a simple example. If students supply some goods 
which are partially public, for example musical instruments, then average parental income may be plausibly assumed to determine the level of such goods, which constitute a contextual effect in our model. Alternatively, data sets exist in which parental involvement in a classroom is measured (e.g. Bassani (2008) and SuiChu and Willms (1996)). The total level of parental involvement can represent a public good analogous to the musical instrument expenditures example. If social units produce public goods, the decision mechanism will implicitly define contextual effects; this occurs in Calabrese et al. (2006).

A second type of partial knowledge of sociomatrices may come from data sets in which individuals are asked to identify those to whom they are connected. These data sets, leaving aside imperfections such as limits on the number of friends that can be named, represent cases in which the analyst has information about connections between individuals but not the sociomatrices themselves. In such contexts, a researcher needs to make a judgment as to the interpretation of the data on direct connections in term of the sociomatrices. Knowledge of the presence or absence of ties between individuals in the network creates a close parallel between identification of social interaction parameters and classical results on the identification of simultaneous equations systems, since holes in the network (i.e. the absence of edges) in essence provide exclusion restrictions that can be exploited.

Throughout subsection 5.1 we impose two additional constraint on models.

E. 6. $-\gamma / \delta$ is not an eigenvalue of $C$.

E. 7. $\phi>0$.

These constraints differ from those in section 4 since they do not pertain to objects observed a priori. Assumption E.6 ensures that $B$ is non-singular. Assumption E.7 is largely for convenience. If $\phi=0$, then the identified matrix $B$ is a linear combination of $I$ and $C$. The converse is true if $I, A, C$ and $A C$ are independent. This is generically true, but we would like to do better since $A$ is unobservable. Results such as corollary 2 show that this can be guaranteed with modest additional a priori information about the peer-effects social network. Such is the case, for example, in theorem 5 below.

5.1. Unknown peer-effects sociomatrix $A$. Peer networks are notoriously hard to measure, and so here we investigate identification when the contextual-effects sociomatrix $C$ is known, but the analyst has either partial information, or none at all, on the peer-effects sociomatrix $A$. For the case in which the researcher knows $C$ and the topology of the peer-effects network, one can establish identification under weak conditions, as seen in theorem 5 .

Theorem 5. Assume that contextual-effects sociomatrix $C$ is known a priori. Assume too that the peer-effects sociomatrix $A$ is unknown but the peer-effects network is known a priori. Suppose that $N \geq 3$. Suppose that there are two distinct 
individuals $j$ and $i$ who are known to be unconnected in the peer-effects network. If $B_{j i}^{-1} \neq 0$, then the utility parameters $\gamma, \delta$ and $\phi$ are identified from the conditional mean of $\omega$ given $x$.

Theorem 5 demonstrates that even when the peer-effects sociomatrix $A$ is unknown, all three utility parameters can still be separately identified from first moments under certain conditions. The theorem presents an instance where the analyst has partial knowledge of $A$. Even without knowledge of $A$, however, identification is possible. Denote by $\mathcal{M}^{\prime}(C)$ the set of models $\mathcal{M}(C)$ satisfying, T.1T.2 and E.1-E.7. Theorem 6 states that the phenomena of which theorem 5 is a specific instance, is generic.

Theorem 6. Assume T.1-T.2 and E.1-E.7. Suppose only the contextual-effects sociomatrix $C$ is known.

i. If $N \geq 3$, then for each $C \in M_{C}$ there is a generic subset $S_{A} \subset M_{A}$ such that if $A \in S_{A}$, then $\gamma, \delta$ and $\phi$ are also identified in $\mathcal{M}_{C}^{\prime}$ by the conditional mean of outcomes given characteristics.

ii. If $E .7$ is relaxed by assuming that $\phi=0$, then result $i$. holds for all but a generic subset of $\mathcal{M}_{C}^{\prime}$.

The theorem specifies conditions under which there exist two distinct (i.e. nonidentical) diagonal elements of $B^{-1}$, which as the proof establishes, is sufficient for identification of all utility parameters. Indeed, under these conditions, the peereffects sociomatrix is also identified. While this may seem surprising, the intuition can be understood by considering equation (4). The dimension of the set of peereffects matrices is $N(N-2)$. The dimension of the set of all $B(s)$ matrices that come from a (known) common contextual-effects matrix $C^{*}$ is no more than $N(N-1)+1$, but we can show it to be no less than $N(N-2)$. We need to recover $N(N-2)+3$ parameters from $B(s)$. Corollary 4 states that this recovery is possible. ${ }^{15}$

Corollary 4. Under the conditions of theorem 6 , the peer-effects sociomatrix $A$ is identified.

5.2. Identification with a priori qualitative network knowledge. As noted in section 2, data sets with network data, such as AddHealth do not furnish the sociomatrices $A$ and $C$ : survey respondents indicate to whom they are connected, but not the weights. We can interpret such data as providing information about exclusion restrictions, the location of 0 's in the $A$ and $C$ matrices. No survey we know of distinguishes between peer- and contextual-effects networks. We suggest that data collection, even if measures of interaction intensity cannot be constructed, allow for distinct sociomatrices.

\footnotetext{
${ }^{15}$ Drton, Foygel, and Sullivant (2011) provide similar results to ours on the recovery of $A$. However, that paper assumes the unobserved heterogeneity is jointly normal and it assumes that contextual effects are absent.
} 
How can such knowledge facilitate identification? In this section we show that if the networks are sufficiently sparse, there is a path to identification which is analogous to classical simultaneous equations results. Without loss of generality, assume that $\mu=0$, since the more general case involves constant terms that are irrelevant to the identification of parameters of interest. The matrix $B$, which characterizes the equilibrium strategy profiles, is identified by theorem 2 , and this identifies the sum $\gamma+\delta$.

Let $b_{i}$ denote the $i$ th row of $B$, and similarly for $A$ and $C$. Consider the $i$ th equation of the system

$$
\begin{aligned}
\omega_{i} & =\frac{\gamma+\delta c_{i i}}{1+\phi} x_{i}+\frac{\delta}{1+\phi} \sum_{j \sim c^{i}} c_{i j} x_{j}+\frac{\phi}{1+\phi} \sum_{j \sim A^{i}} a_{i j} b_{j} \cdot x+\frac{1}{1+\phi} \varepsilon_{i} \\
& =\pi_{1} x_{i}+\sum_{\substack{j \sim C^{i} \\
j \neq i}} \pi_{2 j} x_{j}+\sum_{j \sim{ }_{A} i} \pi_{3 j} b_{j} \cdot x+\frac{1}{1+\phi} \varepsilon_{i}
\end{aligned}
$$

These equations suggest regressing $\omega_{i}$ on the the vectors $x$ and $B x$, and the following theorem describes what can be learned from this procedure. Let $B_{A \neg C}$ denote the submatrix of $B$ whose rows correspond to individuals connected to $i$ through the peer-effects network and whose columns correspond to individuals not connected to $i$ through the contextual-effects network. Recall that $i \sim_{A} j$ indicates that there is an edge between $i$ and $j$ in the peer-effects network, and $i \sim_{C} j$ that an edge exists in the contextual-effects network.

Theorem 7. Assume T.1-T.2 and E.1-E.4. Assume that for some $i, B_{A \neg C}$ has full row rank equal to the number of individuals $j$ connected in the peer-effects network to $i$, denoted as $\#\left\{j: j \sim_{A} i\right\}$. Then

i. The weights $a_{i j}$ for $j \sim_{A} i$ are identified.

ii. $\phi$ is identified.

iii. If there is a $j \neq i$ such that $i \sim_{C} j$, then the point $(\gamma, \delta)$ lies on the line $\gamma+\delta=\beta$ at the point $(\gamma, \delta)=\left(1-c_{i i}\right)^{-1}\left(\beta-\pi_{1}, \pi_{1}-\beta c_{i i}\right)$, for $0 \leq c_{i i}<1$.

iv. If there is a $w \neq i$ such that $i \sim_{C} j$ and $\pi_{2 j}=0$ for some $j \sim_{C} i$, then this is true for all $j \sim_{C} i, \delta=0$ and $\gamma=\pi_{1}$.

$v$. If $k, j \sim_{C} i$ then $c_{i k} / c_{i j}$ is identified.

If $C$ is known a priori then $a_{i}$ (the ith row of $A$ ) and all utility parameters are identified. A necessary condition for these conclusions is that $1+\#\left\{j: j \sim_{C}\right.$ $i, j \neq i\}+\#\left\{j: j \sim_{A} i\right\} \leq N$.

Theorem 7 says that under certain conditions, holes in the network can enable identification: they serve as exclusion restrictions, which can be exploited to back out the utility parameters. Theorem 7's necessary condition is an order condition, and the sufficient condition is a rank condition (see Hsiao (1983, Theorem 3.3.1) for a clear exposition). One way to understand this theorem is to recall that under our assumptions, $B x=\mathrm{E}(\omega \mid x)$. Thus the regression of equation (19) looks 
like the second stage of a two-stage least-squares estimation procedure. The differences between the classical results and ours lay in the fact that in the classical case identification of the second-stage parameter estimates is not an issue, and the rank and order conditions have to do with backing structural parameter estimates out of the second-stage estimates. Here, in contrast, the issue is first identifying the parameters $\pi_{i}$, then using these to recover structural parameters. The utility of part iii. of the theorem is that information about $c_{i i}$ may partially identify $\gamma$ and $\delta$. In particular, if $c_{i i}$ is known a priori, then $\gamma$ and $\delta$ are identified. Finally, note that typically $i \sim_{C} i$, and in this case the necessary condition is that the total number of edges emanating from $i$ in either network not exceed $N$.

The results in sections 5.1 and 5.2 have important implications in terms of the interpretation of surveys that measure social networks. In terms of interpretation, Theorems 5-7 demonstrate the importance of network structure in generating identification. The key conditions across our results is a priori knowledge of 0's in the sociomatrices. Survey data on social networks do not provide information on the intensity of bilateral interactions. Rather they provide information on whether or not a bilateral interaction is present. Our emphasis on the importance of "holes" in the social structure extends the argument in Bramoullé, Djebbari, and Fortin (2009) that 0's in a known sociomatrix allow for instruments. Our results show that these 0's can facilitate identification even when, unlike Bramoullé, Djebbari and Fortin, the sociomatrices are unknown.

Our results also suggest a potentially serious limitation in current surveys, specifically Addhealth, which is arguably the most popular data set for the study of social network effects. Its main draw is that high school students in its nationally representative sample are interviewed not only about the usual demographic and outcome variables of interest, but also about who their friends are. Unfortunately, the data set's friendship questions are restricted in that each student is allowed to name up to 5 friends of each gender. This has important ramifications in view of the result in theorem 5, which indicates that it is more useful to know who is not someone's friend rather than who is. Moreover, the restriction on the number of friends means that the failure to identify someone as a friend does not mean that there is a corresponding zero in the associated sociomatrices. While the limitation on the number of friends that could be named in the interviews has long been understood as inducing measurement error in network structure, as far as we know, the effects of this limitation on identification per se have not been recognized. ${ }^{16}$

Our results provide an important generalization of Lee, Liu, and Lin (2010) as we do not need to assume that each agent equally weights others to whom he is directly connected. Lee, Liu, and Lin assume that the sociomatrices are functions of the common adjacency matrix, which clearly does not need to be the case.

\footnotetext{
${ }^{16}$ Another concern is that the failure to identify someone as a friend is consistent with a negative entry in one or both of the sociomatrices we have employed. While we have assumed that all elements of $A$ and $C$ are non-negative (axiom T.1) negative values are certainly empirically plausible. We thank Jesse Naidoo for this observation
} 
5.3. Identification with aggregated social network data. We conclude this section with an analysis of a different type of partial knowledge, namely partial knowledge that reflects the absence of individual level data with which to evaluate social effects. Section 5.2 provided some positive results on inference of structural parameters when social interaction effects data are aggregated. Here we show that these effects disappear when individuals are sampled across groups and paired with group level averages. For data sets employing the PSID, for example, it is common to see models in which individual outcomes are assumed to depend on individual characteristics and certain census tract aggregates. We provide a link to this type of empirical analysis by considering the case where data are of the form $\omega_{i}, x_{i}, \bar{\omega}^{g}, \bar{x}^{g}$, where $\bar{\omega}^{g}$ and $\bar{x}^{g}$ denote group level average outcomes and characteristics, respectively, of $i$ 's group $g$. We make two knowledge assumptions. First, we assume that $C$ is known because otherwise information would be lost relative to theorem 2 and identification would obviously fail. Second, the analyst only observes one individual per group, whom we denote as 1 .

K. $1^{\prime \prime} . C$ is exogenous and known to the analyst.

K. $2^{\prime \prime}$. For all $g$, the analyst observes $\left(\bar{\omega}^{g}, \bar{x}^{g}, \omega_{1}, x_{1}\right)$.

Finally, we place a restriction on the nature of observed heterogeneity, namely, that it is i.i.d. across members of the same group.

E. 8. For each $g, x_{i}$ is i.i.d. within $g$.

For each individual $i$ in the sample we observe that individual's record and his group averages. The presumption is that the individual's social network is confined to the group. The individual can be netted out of the group average, so from equation (5) we derive two relationships: one for the behavior of everyone but individual $i$, and one for the behavior of individual $i$.

$$
\begin{aligned}
\mathrm{E}\left(\bar{\omega}^{g} \mid x_{i}, \bar{x}^{g}\right) & =\mu^{g}+b^{g} \mathrm{E}\left(x \mid x_{i}, \bar{x}^{g}\right)+b_{g i} x_{i} \\
\mathrm{E}\left(\omega_{i} \mid x_{i}, \bar{x}^{g}\right) & =\mu_{i}+b_{-i} \mathrm{E}\left(x \mid x_{i}, \bar{x}^{g}\right)+b_{i i} x_{i}
\end{aligned}
$$

where the bars denote group averages exclusive of individual $i$, and variables with a $i$ subscript refer to individual $i$. The coefficients $b_{g i}=\sum_{k \in g, k \neq i} b_{k i}, b^{g}=$ $(1 /(N-1)) \sum_{j, k \in g, k \neq i} B_{k j}$, and $b_{-i}=\sum_{j \in g, j \neq i} b_{i j}$ are all sums of terms in the matrix $B$.

Theorem 8. Assume T.1-T.2, E.1-E.4 and E.8, and suppose that $\mathrm{E}\left(\bar{\omega}^{g} \mid x_{i}\right)$ and $\mathrm{E}\left(\omega_{i} \mid x_{i}, \bar{x}^{g}\right)$ are known. Then $\beta=\gamma+\delta$ is identified, and $\gamma, \delta, \phi$ are not identified.

Theorem 8 shows that the assumption of a linear-in-means structure entails too great a loss of information to allow for identification of the utility parameters. As in other cases, if the projection of $\omega_{i}$ onto $x_{i}$ and $x_{-i}$ differs from the projection of $\omega_{i}$ onto $x_{i}$, then all one can say is that some sort of social interaction is present. 
This is a cautionary message given the ubiquity of these models in empirical practice. Our results provide a complement to Davezies, d'Haultfoeuille, and Fougère (2009) who consider the problem of identification for a linear-in-means model in which the analyst does not have data on the group aggregate variables, but does know the group sizes. Identification is shown to hold when there are groups of at least 3 distinct sizes. Our relatively negative result stems from the heterogeneity in sociomatrices across groups. This precludes our use of that paper's approach, whereby observed means of others can be treated as mismeasured true means. For our context, the mismeasurement involves loss of information on the weights of the sociomatrix as well as the values of $x$ and $\omega$.

\section{ENDOGENEITY OF SOCIAL StRUCTURE}

In this section we explore the consequences of network endogeneity. We first discuss the self-selection problem. Second, we examine how durable networks, in which unobserved (to the analyst) determinants of social interactions arise after the network is formed, create a distinct identification issue.

6.1. The selection problem. A standard concern in uncovering social interactions is the endogeneity of the social structure. The issue is straightforward: does a correlation between high ability friends and an individual student's educational performance reflect a social interaction of the type we have modeled or does it occur because the student's unobserved type is correlated with his friendship choices? This concern has generated interest in randomized assignment to groups, as in Sacerdote (2001), as well as cases in which a "natural experiment" alters group composition, e.g. Cipollone and Rosolia (2007). ${ }^{17} \mathrm{~A}$ focus on data in which exogenous social structure is present delimits the domain of environments that may be studied, so it is important to understand how endogeneity should be understood and accounted for in more general settings.

A natural way to extend our model of social interactions to network formation is to formulate a two-stage game, in which networks are formed in the first stage and actions are determined in the second. For each possible network there is a unique second-stage equilibrium, and each individual's expected utility of this second-stage equilibrium is a value function for the network which gives payoffs for the first-stage game.

While this abstract conceptualization is useful in understanding the implications of endogeneity, it is not one that can be directly implemented in the context of an econometric model of network formation and subsequent choices. The reason for this is that there simply does not exist a viable general theoretical model of network formation. Networks for business relations, job search and classroom

\footnotetext{
${ }^{17}$ Blume et al. (2011) discuss how quasi-experiments may not satisfactorily resolve self-selection problems in identifying social interactions.
} 
friendships are formed according to very different rules, and vary greatly in the degree to which they are instrumental for the second-stage game. While network formation games have been formulated for particular contexts, they do not even include pair-specific weights in the decision process.

An alternative approach is to imagine conditions that should be properties of equilibrium outcomes for many different games. This path, first travelled by Gale and Shapley (1962), leads to network stability concepts such as pairwise stability (Jackson and Wolinsky 1996) and pairwise-Nash stability (Calvó-Amrengol and Ilkiliç 2009). A network is pairwise-Nash stable if and only if a) no individual wants to drop any edges, and $b$ ) there is no missing edge that if added would, ceteris paribus, be a Pareto improvement for the individuals it connects. It is neither a strictly cooperative nor a strictly non-cooperative concept. Stability expresses the idea that breaking relations is a non-cooperative activity while forming new relations involves mutual consent.

While this approach has been employed in a few recent studies ${ }^{18}$ it is not a panacea. The basic problems are threefold. First, stable networks may not exist. Non-existence, however, can be circumvented by introducing random stable networks, that is, probability distributions on graphs which satisfy an expectation based concept of stability - that is, one can imagine a probability distribution on graphs for which the inequalities in the stability definition are satisfied in expectation. Existence can easily be shown in two cases: if $\varepsilon$ is observed only just prior to the second stage or the support of the marginal distribution of $\varepsilon$ is finite. ${ }^{19}$ In the first case, selection is not an issue because private types are not observed until after the network is formed. In the second case, discreteness of the set of possible z's rules out many common econometric models. It is quite possible, however, that an existence proof can be provided for more general classes of models.

A second problem for both pairwise-stable and random pairwise-stable networks is that factors other than the utility of second-stage choices may play a role in determining the utility of a given network. The sociology literature is replete with descriptions of such payoffs. For instance, there might be an independent value to homophily - associating with people similar to oneself — which is distinct from

\footnotetext{
${ }^{18}$ Badev (2013) studies the coevolution of friendship networks and smoking behaviors in an environment where agents make myopic friendship decisions among $k-1$ randomly selected others. This model is shown to converge to a $k$-stable Nash network, which means that no agent wishes to deviate by simultaneously altering $k-1$ friendship statuses as well as his choice. Sheng (2012) uses pairwise stability in the context of identification of a network formation game but omits choices that are affected by network structure. Hsieh and Lee (2012) follow a strategy related to ours but with perfect information, considering a two-stage game in which social structure is formed in stage 1 and choices are made in stage 2.

${ }^{19}$ For both cases, consider Myerson's (1991) network formation game. In the first case, the firststage game is a complete-information game, and a correlated equilibrium will satisfy the needed inequalities. In the second case, a perfect direct correlated equilibrium (Dhillon and Mertens 1996) of Myerson's (1991) network formation game is a pairwise-Nash stable random graph, and since Myerson's game is finite, these equilibria exist.
} 
the value of the game outcome. Structural estimation of these models, then, involves specifying these additional factors. This requirement may be impossible to realize.

A third problem is that the set of pairwise stable random graphs will typically not be a singleton. Thus partial-identification techniques will come into play, and it may be that the set of pairwise stable random graphs is too large to impose useful first-stage restrictions.

For these reasons, we believe it makes more sense to address endogeneity by considering its effects on inference from data on the second stage of the game. This involves returning to our model and asking how endogeneity can invalidate our assumptions. From this vantage point, the implications of endogeneity depend on the information available to agents when networks form.

If either the public types or the private types relevant for the second-stage choice are not observed at the time the network is formed, then the missing variable cannot enter into the first-stage interim payoff functions. In this case, the linear structure of the second stage is maintained and endogeneity is not an issue. By contrast, suppose that $x$ and $z_{i}$ are available to agent $i$ at the outset of the first stage. The expected second state payoff will depend upon both of these variables, and so both will influence individuals' first-stage choices. Consequently, an individual $i$, observing that he is connected to $j$, can, with knowledge of $x_{j}$, make an inference about the value of $z_{j}$ that is dependent on $x_{j}$. Thus E.4 is violated. In this case $\mu(x, z)$ is not independent of $x$, and second-stage equilibrium strategy profiles are no longer linear in $x$, except for special cases. (They are, however, still described by theorem 1.) This is the selection problem. It is not just a statistical issue. It affects the basic structure of equilibrium, because it affects inference not only of the econometrician but of individuals constructing the network.

How can one proceed? From the perspective of the reduced form model (5) coefficients $B$, the only effect that endogeneity can have under the information regime we have described is through $\mathrm{E}(\varepsilon \mid x)=\mathrm{E}(\mu(x, z) \mid x)$. This expression is in fact nothing more than Heckman's classic control function (e.g. Heckman (1979), Heckman and Robb (1986)). So long as $\mu(x, z)$ does not depend linearly on $x$, identification will still hold. To be clear, the robustness of identification to endogenous network formation exploits the quadratic game structure that leads to linear equilibrium strategy profiles. But this is true for general control function approaches; they break down when $\mathrm{E}(\varepsilon \mid x)$ is linear in $x$. Hence Heckman's fundamental idea that self-selection can be addressed by incorporating self-selection into the analysis, rather than using instrumental variables, applies to social interactions contexts. $^{20}$

\footnotetext{
${ }^{20}$ The idea that selection on unobservables can aid in identification of social effects via control functions was first shown in Brock and Durlauf (2001). Brock and Durlauf (2006) provide a more general treatment when agents select into non-overlapping groups and the sociomatrix weights are required to be equal as occurs for the linear-in-means model. Our current discussion makes two
} 
Where would instrumental variables approaches come into play in this setting? Suppose that the researcher has available a vector of observable individual attributes $v$. From the vantage point of this two-stage game, the critical question involves the timing by which this information is revealed. If agents observe $v$ by the outset of the second stage, then endogenous network formation means that one needs to analyze $\mathrm{E}\left(z_{i} \mid x, v\right)$. But this means that $v$ no longer constitutes an instrument, since it is correlated with the errors in the regressions that emerge in the second stage of the game. In this sense, the pro forma use of instruments on the grounds that they are associated with the payoffs of network formation and not behaviors conditional on the network is invalid. Once one introduces instruments to account for network heterogeneity, one needs to account for their implications for the second stage regression errors, which will, outside of special cases, be present even if the payoff in the second stage is independent of the instrument.

6.2. Durable Networks. The preceding discussion took the point of view that network formation transpired prior to, and only for the purpose of, second-stage strategic interaction. An alternative (and sociologically richer) view is that a given social network serves many purposes, and co-evolves continuously with the behaviors it facilitates. We submit that for many of the subjects to which social interaction models have been applied, this is more descriptively accurate than the preceding two-stage game. Suppose, then, that social networks are durable informal institutions, and that relations are ongoing. It is reasonable to suppose that as a result of this process, variables that are unobserved at the time of network formation are revealed over time to network members and generate social interactions, but nonetheless may remain invisible to the researcher. Such variables may include things like generalized ability, or personality traits that the researcher cannot measure but may nonetheless theorize about. These variables - public to network members but unobservable to the analyst - leave traces in the correlation of individuals' choices. In our view, the issue of unobserved carriers of social interactions represents the complement to the standard concern about self-selection that is the focus of Section 6.1. Endogeneity of networks, we argue, can occur with respect to one set of variables, while social interactions occur with respect to another. Endogeneity, from this vantage point, alters the relationship between the variables relevant to the second stage of the game and the variables relevant to the first. In this subsection we investigate what can be learned about utility parameters for such variables from second moments.

We suppose that there is a characteristic $u$ which is unobservable to the econometrician, but observable to individuals in the networks. We amend the theory

important extensions of this earlier work. First, an explicit game for the sequential formation of social networks and the subsequent choices of actors in the network are described. Second, the analysis indicates that the control function approach applies to a much wider class of environments than had previously been established. 
accordingly, so the utility function is now ${ }^{21}$

$$
\begin{aligned}
U_{i}\left(\omega_{i}, \omega_{-i}\right)=\left(\gamma x_{i}+\delta \sum_{j} c_{i j} x_{j}+\gamma_{u} u_{i}\right. & \left.+\delta_{u} \sum_{j} c_{i j} u_{j}+z_{i}\right) \omega_{i} \\
& -\frac{1}{2} \omega_{i}^{2}-\frac{\phi}{2}\left(\omega_{i}-\sum_{j} a_{i j} \omega_{j}\right)^{2}
\end{aligned}
$$

The equilibrium analysis is unchanged, and the equilibrium strategy profile is

$$
\begin{aligned}
f(x, u, z) & =\frac{1}{1+\phi}\left(I-\frac{\phi}{1+\phi} A\right)^{-1}(\gamma I+\delta C) x \\
& +\frac{1}{1+\phi}\left(I-\frac{\phi}{1+\phi} A\right)^{-1}\left(\gamma_{u} I+\delta_{u} C\right) u+\mu(x, u, z)+\frac{1}{1+\phi} z
\end{aligned}
$$

where $\mu(x, u, z)$ is derived in a fashion analogous to $\mu(x, z)$. We make the following assumptions about observations:

K. $1^{\prime \prime \prime}$. A and $C$ are exogenous and known to the analyst a priori.

K. $2^{\prime \prime \prime}$. For all $i$, the analyst observes $\left(\omega_{i}, x_{i}\right)$, but not $u_{i}$.

Under these assumptions, the econometric model becomes

$$
\omega=\mu+B(s) x+B_{u}(s) u+\varepsilon
$$

where

$$
B_{u}(s)=B_{\phi}(s)\left(\gamma_{u} I+\delta_{u} C\right) .
$$

The unobserved component $\varepsilon$ is now the entire second line of equation (22).

We ask two questions in this framework: when are the utility parameters $\gamma$, $\delta$ and $\phi$ identified from $B(s)$, and when are the utility parameters corresponding to the unobserved public variables $\gamma_{u}$ and $\delta_{u}$ identified? The answer to the first question is straightforward: if $(u, z)$ is independent of $x$, then $\mu(x, u, z)$ will be independent of $x$, and so all the theorems above not requiring independent errors still apply.

More interesting is the second question, what can be identified concerning utility parameters for the unobservable public type. While social interactions effects pertaining to unobservables have yet to be systematically investigated, there are good reasons to think that such effects are of interest. For example, in the modern work on cognitive and noncognitive skills, e.g. Cunha and Heckman (2007) and Cunha, Heckman, and Schennach (2010), these skills are explicitly interpreted as latent variables in a measurement system. It is natural to think that the skills of others affect a student in a classroom, for example. An important extension of our

\footnotetext{
${ }^{21}$ We have assumed the same sociomatrix describes contextual effects for both $x$ and $u$. The cost of relaxing this assumption is that the econometrician is required to know a priori both sociomatrices.
} 
result would involve an explicit consideration of unobservables when a measurement system exists that relates the unobservables to observables such as test scores. Identification will have to come from the covariance matrix, and this will require strong independence hypotheses. We extend our econometric assumptions as follows:

E. $4^{\prime}$. For all $k, i, j \in V, x_{k}, u_{i}$ and $\varepsilon_{j}$ are pairwise independent.

It follows from E.4' that equation (2a) still holds, and that the $u_{i}$ will be independent. We have the following result:

Theorem 9. Suppose that the $\varepsilon_{i}$ are i.i.d. with variance $\sigma_{\varepsilon}^{2}$, that the $u_{i}$ are i.i.d. with variance $\sigma_{u}^{2}$, and that assumptions T.1-T.2, E.1-E.3, E.4', E.5, and K.1'1'-K.2'"'. For generic pairs $(A, C)$ of peer- and contextual-effects sociomatrices, the following are identified from the covariance matrix of $\omega: \gamma, \delta, \phi$, and $\sigma_{\varepsilon}^{2}$, and the sets $\pm\left(1 / \sigma_{u}^{2}\right)\left(\gamma_{u}, \delta_{u}\right)$. In particular, the sets $\gamma_{u}=0$ and $\delta_{u}=0$, and the ratio $\gamma_{u} / \delta_{u}$ (when it is defined) are identified.

The parameters $\gamma_{u}$ and $\delta_{u}$ are identified only up to a non-zero multiple, $\pm \sigma_{\epsilon}^{2}$, but this nonetheless identifies the ratio $\gamma_{u} / \delta_{u}$. Identifying this ratio is useful because we learn the relative significance of own versus contextual effects. Identification up to a sign is also interesting because we may have a priori information that signs one of the terms. For instance, in studies of earnings in workplace networks, ability has both an own effect and a contextual effect. While the sign of the own effect may be obvious, the sign of the contextual effect will depend upon whether workers and worker tasks are substitutes or complements in the production process.

It is possible to give conditions on edges in the network that imply the $u_{i}$ independence condition, just as was done for the $\varepsilon_{i}$-independence condition in section 4 . We also conjecture that one can go further in identifying the magnitudes of $\gamma_{u}$ and $\delta_{u}$. Let $T$ denote the transpose of a matrix. Since $\phi /(1+\phi)$ and $\sigma_{\varepsilon}^{2}$ are identified, the econometrician can observe

$$
\begin{aligned}
(1+\phi)^{2} B_{u} u \cdot\left(B_{u} u\right)^{T}=\sigma_{u}^{2}(I-\phi & \left.(1+\phi)^{-1} A\right)^{-1}\left(\gamma_{u} I+\delta_{u} C\right) \\
& \cdot\left(\gamma_{u} I+\delta_{u} C^{T}\right)\left(I-\phi(1+\phi)^{-1} A^{T}\right)^{-1} .
\end{aligned}
$$

The difficulty in pinning down magnitudes of $\gamma_{u}$ and $\delta_{u}$ is the presence of $\sigma_{u}^{2}$. Suppose, however, that the components of the contextual-effects network $\mathcal{C}$ are unions of components of the peer-effects network $\mathcal{A}$. This is the setting of section 4.2. Suppose $\mathcal{C}$ has $H$ such components. Index all symbols by $h$, the component to which they apply, and let $\tilde{n}^{h}$ denote the row vector with all terms $1 / n^{h}$ and of length $n^{h}$, the size of component $h$. Then following Graham (2008), ratios of the form

$$
\tilde{n}^{h} B_{u}^{h} u^{h} \cdot\left(B_{u}^{h} u^{h}\right)^{T}\left(\tilde{n}_{t}^{h}\right)^{T} / e^{1} B_{u}^{1} u^{1} \cdot\left(B_{u}^{1} u^{1}\right)^{T}\left(e_{t}^{1}\right)^{T}
$$


are independent of the variance of $\omega$. This is essentially a variance contrast method. One can show with a transversality argument like that in the proof of theorem 4, that if $\mathcal{C}$ has at least 4 components, then for any $A$ and generic $C$, $\pm\left(\delta_{u}, \sigma_{u}\right)$ will be identified. The sign ambiguity remains because both the numerator and denominator are linear combinations of products of degree 2 .

Of course, unobserved public variables are not the only source of correlated shocks across individuals. Bramoullé, Djebbari, and Fortin (2009) consider the problem of group shocks, a common shock that effects $i$ and everyone connected to her. They provide an identification criterion like our theorem 3 when $A=C$ involving independence of matrix products up to third order, which comes from a procedure in which each individual $i$ 's behavior is differenced from the average behavior of her neighbors. Bramoullé, Djebbari, and Fortin's insight that de-meaning individual choices using group level averages can eliminate group-specific fixed effects, is complementary to other forms of differencing. As a whole, appropriate choice of differencing can eliminate the role of group effects, which is why their presence has not been a focus of this paper. ${ }^{22}$

\section{CONCLUSION}

In this paper, we have provided a theoretical and econometric characterization of linear social interactions models. Our analysis provides both a clear description of the behavioral assumptions needed to employ these models as well as the conditions under which the primitive utility parameters that characterize social influences may be recovered. Our results demonstrate the possibilities and limits to identification as determined by the degree of prior information on the sociomatrices that determine how the characteristics and behaviors of others affect each individual's utility. We show that for the most common case in the empirical literature, namely when these matrices are known a priori identification holds generically. The absence of any a priori knowledge on these matrices unsurprisingly means that identification fails. The known sociomatrices results demonstrates that variants of the workhorse linear-in-means model for which identification fails are in fact knife edge cases.

We further explore a range of possible forms of a priori knowledge that represent intermediate cases compared to these two extreme information assumptions. These intermediate cases correspond to plausible source of a priori information as derived from economic theory and/or empirical social structure measurement. We also address the identification question when a researcher is limited to aggregated data of various types. Finally, we demonstrate that endogenous network formation does not constitute an unbridgeable impediment to identification.

\footnotetext{
${ }^{22}$ Kwok (2012) explores the effects of alternate differencing schemes on identification. He shows that different schemes can affect the information in the de-meaned data. Hence the choice of demeaning procedure matters.
} 
In terms of future research, we see a number of important directions. First, our findings may be understood as fleshing out parts of the "assumptions/possibilities" frontier in terms of the edges between different types of a priori information on social structure and identification. There is no reason to believe that the cases we have examined span the possible types of information that may be available to a researcher, so there is certainly more work to be done in fully characterizing the environments in which identification does or does not hold.

Second, the operationalization of the control function approach to addressing network endogeneity needs to be developed. Third, we have not addressed issues of estimation. This suggests a necessary complementary paper to this one if one wishes to make our results operational. For example, the positive identification results we provide on endogenous social structure formation need to be supplemented with procedures for constructing the analog to control functions whose existence are implicit in the identification theorem.

Fourth, while we have addressed the question of how our identification results are affected by endogenous social structure, we have not addressed how this endogeneity can, when explicitly modeled, facilitate identification. For example, if group memberships are associated with prices, then prices can help to uncover social effects, as demonstrated in recent advances in the econometrics of hedonic models. (See Ekeland, Heckman, and Nesheim (2004)). As discussed above, the control functions associated with the changes in conditional error distributions conditional on group membership may be able to facilitate identification.

Finally, information on social interactions may be encoded in the composition of the groups themselves. Becker's (1957) model of taste-based discrimination implies that information on the presence of discriminatory preferences is embodied both in any black/white wage gap and in the degree of segregation of workers across firms. All these directions emphasize the importance of extending the theoretical and econometric arguments developed here in directions that fully exploit the codetermination of social structure and associated behavioral outcomes. 


\section{REFERENCES}

Badev, A. 2013. "Discrete Games with Endogenous Networks: Theory and Policy." Working Paper. University of Pennsylvania.

Bassani, C. 2008. "Parent Classroom Involvement and the Development of Social Capital: A Reading Program in East Vancouver." Journal of Educational Enquiry 8 (2):51-70.

Bayer, P., S. Ross, and G. Topa. 2008. "Place of Work and Place of Residence: Informal Hiring Networks and Labor Market Outcomes." Journal of Political Economy 116 (6):1150-1196.

Becker, G. 1974. "A Theory of Social Interactions." Journal of Political Economy $82(6): 1063-1093$.

Benhabib, J., A. Bisin, and M. Jackson. 2011a. Handbook of Social Interaction, vol. 1. Amsterdam: North Holland.

. 2011b. Handbook of Social Interaction, vol. 2. Amsterdam: North Holland.

Bifulco, R., J. Fletcher, and S. Ross. 2011. "The Effect of Classmate Characteristics on Post-secondary Outcomes: Evidence from the Add Health." American Economic Journal: Economic Policy 3 (1):25-53.

Blume, L., W. Brock, S. Durlauf, and Y. loannides. 2011. "Identification of Social Interactions." In Handbook of Social Economics, vol. 1B, edited by J. Benhabib, A. Bisin, and M. Jackson. Amsterdam: North Holland, 853-964.

Bochnak, J., M. Coste, and M-F. Roy. 1987. Geometrie Algebrique Reele. Berlin: Springer-Verlag.

Bramoullé, Y., H. Djebbari, and B. Fortin. 2009. "Identification of Peer Effects Through Social Networks." Journal of Econometrics 150:41-55.

Brock, W. and S. Durlauf. 2001. "Interaction-Based Models." In Handbook of econometrics, vol. 5, edited by J. Heckman and E. Leamer. Amsterdam: NorthHolland, 3297-3380.

- 2006. "Multinomial Choice with Social Interactions." In The Economy as an Evolving Complex System, Vol. 3, edited by S. Durlauf and L. Blume. New York: Oxford University Press, 175-206.

Calabrese, S., D. Epple, T. Romer, and H. Sieg. 2006. "Local Public Good Provision: Voting, Peer Effects, and Mobility." Journal of Public Economics 90 (6):959-981.

Calvó-Amrengol, A. and R. Ilkiliç. 2009. "Pairwise-Stability and Nash Equilibria in Network Formation." International Journal of Game Theory 38:51-79.

Calvó-Armengol, A., E. Patacchini, and Y. Zenou. 2009. "Peer Effects and Social Networks in Education." Review of Economic Studies 76:1239-1267.

Cipollone, P. and A. Rosolia. 2007. "Social Interactions in High School: Lessons from an Earthquake." The American Economic Review 97 (3):948-965.

Conley, T. and C. Udry. 2010. "Learning About a New Technology: Pineapple in Ghana." American Economic Review 100 (1):35-69.

Corcoran, M., R. Gordon, D. Laren, and G. Solon. 1992. "The Association between Men's Economic Status and their Family and Community Origins." Journal of Human Resources 27 (4):575-601. 
Cunha, F. and J. Heckman. 2007. "The Technology of Skill Formation." The American Economic Review 97 (2):31-47.

Cunha, F., J. Heckman, and S. Schennach. 2010. "Estimating the Technology of Cognitive and Noncognitive Skill Formation." Econometrica 78 (3):883-931.

Datcher, L. 1982. "Effects of Community and Family Background on Achievement." Review of Economics and Statistics 64:32-41.

Davezies, L., X. d'Haultfoeuille, and D. Fougère. 2009. "Identification of Peer Effects Using Group Size Variation." The Econometrics Journal 12 (3):397413.

De Giorgi, G., M. Pellizari, and S. Redaelli. 2010. "Identification of Social Interactions through Partially Overlapping Peer Groups." American Economic Journal: Applied Economics 2:241-275.

Dhillon, A. and J.-F. Mertens. 1996. "Perfect Correlated Equilibria." Journal of Economic Theory 68:279-302.

Drton, M., R. Foygel, and S. Sullivant. 2011. "Global Identifiability of Linear Structural Equation Models." Annals of Statistics 39 (2):865-86.

Ekeland, I., J. Heckman, and L. Nesheim. 2004. "Identification and Estimation of Hedonic Models." Journal of Political Economy 112 (1-2).

Epple, D. and R. Romano. 1998. "Competition Between Private and Public Schools, Vouchers, and Peer-Group Effects." The American Economic Review $88(1): 33-62$.

- 2011. "Peer effects in education: A survey of the theory and evidence." In Handbook of Social Economics, vol. 1a, edited by J. Benhabib, A. Bisin, and M. Jackson, chap. 11. Amsterdam: North Holland, 1053-1163.

Fisher, F. 1966. The Identification Problem in Econometrics. New York: McGrawHill.

Gale, D. and L. Shapley. 1962. "College Admissions and the Stability of Marriage." American Mathematical Monthly 69:9-15.

Glaeser, E., B. Sacerdote, and J. Scheinkman. 1996. "Crime and Social Interactions." Quarterly Journal of Economics 111:507-48.

—. 2003. "The Social Multiplier." Journal of the European Economic Association 1:345-53.

Graham, B. 2008. "Identifying Social Interactions Through Conditional Variance Restrictions." Econometrica 76 (3):643-60.

Granovetter, M. S. 1973. "The Strength of Weak Ties." American Journal of Sociology 78 (6):1360-80.

Heckman, J. 1979. "Sample Selection Bias as a Specification Error." Econometrica 47:153-161.

Heckman, J. and R. Robb. 1986. "Alternative Methods for Solving the Problem of Selection Bias in Evaluating the Impact of Treatments on Outcomes." In Drawing inferences from self-selected samples, edited by $\mathrm{H}$. Wainer. New York: Springer-Verlag, 63-113.

Henderson, Vernon, Peter Mieszkowski, and Yvon Sauvageau. 1978. "Peer Group Effects and Educational Production Functions." Journal of Public Economics $10(1): 97-106$. 
Hsiao, C. 1983. "Identification." Handbook of econometrics 1:223-283.

Hsieh, C.-S. and L.-F. Lee. 2012. "A Structural Modeling Approach for Network Formation and Social Interactions with Applications to Students' Friendship Choices and Selectivity on Unobservables." Working Paper. Ohio State University.

Jackson, M. and A. Wolinsky. 1996. "A Strategic Model of Social and Economic Networks." Journal of Economic Theory 71 (1):44-74.

Koopmans, T. 1953. "Identification Problems in Economic Model Construction." In Studies in Econometric Method, edited by William C. Hood and Tjalling C. Koopmans. New York: Wiley and Sons, 27-48.

Kwok, H. 2012. "Identification Problems of Linear Social Interaction Models: A General Analysis Based on Matrix Spectral Decompositions." Mimeo.

Lee, L.-F. 2007. "Identification and Estimation of Econometric Models with Group Interactions, Contextual Factors and Fixed Effects." Journal of Econometrics 140:333-74.

Lee, L.-F., X. Liu, and X. Lin. 2010. "Specification and Estimation of Social Interaction Models with Network Structure, Contextual Factors, Correlation and Fixed Effects." Econometrics Journal 13 (2):145-176.

Lin, N. 2002. Social Capital: A Theory of Social Structure and Action, vol. 19. Cambridge University Press.

Manski, C. 1993. "Identification of Endogenous Social Effects: The Reflection Problem." Review of Economic Studies 60:531-42.

Manski, C. and J. Mayshar. 2003. "Private Incentives and Social Interactions: Fertility Puzzles in Israel." Journal of the European Economic Association $1(1): 181-211$.

McManus, D. 1992. "How Common is Identification in Parametric Models?" Journal of Econometrics 53 (1):5-23.

Moffitt, R. 2001. "Policy Interventions, Low-Level Equilibria, and Social Interactions." In Social dynamics, edited by Steven N. Durlauf and H. Peyton Young. Cambridge MA: MIT Press, 45-82.

Montgomery, J. 1994. "Weak Ties, Employment, and Inequality: An Equilibrium Analysis." American Journal of Sociology 99:1212-36.

Myerson, R. 1991. Game Theory: Analysis of Conflict. Cambridge MA: Harvard University Press.

Nakajima, R. 2007. "Measuring Peer Effects on Youth Smoking Behaviour." Review of Economic Studies 74 (3):897-935.

Patacchini, E., E. Rainone, and Y. Zenou. 2012. "Student Networks and Long-Run Educational Outcomes: The Strength of Strong Ties." CEPR Discussion Paper No. DP9149.

Rege, M., K. Telle, and M. Votruba. 2012. "Social Interaction Effects in Disability Pension Participation: Evidence from Plant Downsizing." The Scandinavian Journal of Economics 114:1208-1289.

Sacerdote, B. 2001. "Peer Effects with Random Assignment: Results for Dartmouth Roommates." Quarterly Journal of Economics 116:681-704. 
2011. "Peer Effects in Education: How Might They Work, How Big are They and How Much Do We Know Thus Far?" Handbook of the Economics of Education 3:249-277.

Sharkey, P. and F. Elwert. 2011. "The Legacy of Disadvantage: Multigenerational Neighborhood Effects on Cognitive Ability." AJS; American journal of sociology 116 (6):1934-1981.

Sheng, S. 2012. "Identification and Estimation of Network Formation Games." Working Paper. University of Southern California.

Sirakaya, S. 2006. "Recidivism and Social Interactions." Journal of the American Statistical Association 101 (475):863-877.

Sui-Chu, E. and J. Willms. 1996. "Effects of Parental Involvement on Eighth-Grade Achievement." Sociology of education :126-141.

Topa, G. 2001. "Social Interactions, Local Spillovers and Unemployment." The Review of Economic Studies 68 (2):261. 


\section{APPENDIX}

In fact we prove a more general theorem. Suppose that each individual $i$ has his or her own $\phi_{i}$. Define the matrices

$$
\Phi_{i j}=\left\{\begin{array}{ll}
\frac{1}{1+\phi_{i}} & \text { if } i=j, \\
0 & \text { otherwise; }
\end{array} \quad \hat{\Phi}_{i j}= \begin{cases}\frac{\phi_{i}}{1+\phi_{i}} & \text { if } i=j, \\
0 & \text { otherwise. }\end{cases}\right.
$$

Assumption T.1 is modified appropriately:

T. $1^{\prime}$. For all $i, \phi_{i} \geq 0$. $A$ and $C$ are non-negative, for each $i \in V, \sum_{j} a_{i j}$ is either 0 or 1 , and similarly for $C$. For all $i \in V, a_{i i}=0$.

Theorem A1. If the Bayesian game satisfies axioms T.1' and T.2, then the game has a unique Bayes-Nash equilibrium. The equilibrium strategy profile is

$$
f(x, z)=\Phi(I-\hat{\Phi} A)^{-1}(\gamma I+\delta C) x+g(x, z),
$$

where $g(x, z)$ satisfies, for each $i$, the relation

$$
g_{i}\left(x, z_{i}\right)=\frac{1}{1+\phi_{i}} z_{i}+\frac{\phi_{i}}{1+\phi_{i}} \sum_{j} a_{i j} \mathrm{E}\left(g_{j}\left(x, z_{j}\right) \mid x, z_{i}\right) .
$$

If $z$ is independent of $x$, then each $g\left(x, z_{i}\right)$ depends only on $z_{i}$. If the elements of $z$ are pairwise independent, then $g_{i}\left(x, z_{i}\right)=\left(1+\phi_{i}\right)^{-1} z_{i}+\mu_{i}(x)$.

This theorem breaks the strategy profile into two pieces. The first measures direct and contextual effects of the public type $x$, and the feedback through their peer effects. The second term measures the effects of each individual's private type and is estimate of the private types of others.

Proof of Theorem A1. Suppose that in the payoff function (1) the parameter $\phi$ is indexed by $i$. Give $\mathcal{F}$ the $L_{\rho}^{2} \max$ norm; $\|f\|=\max _{i}\left\|f_{i}\right\|_{2}$. Let

$$
\psi_{i}=\gamma x_{i}+\delta \sum_{j} c_{i j} x_{j}+z_{i}
$$

so that

$$
u_{i}=\psi_{i} \omega_{i}-\frac{1}{2} \omega_{i}^{2}-\frac{\phi_{i}}{2}\left(\omega_{i}-\sum_{j} a_{i j} \omega_{j}\right)^{2}
$$

Since the strategies are in $L_{\rho}^{2}$, the expected payoff to any $i$ of any strategy profile $f$ is finite, so preferences over strategies for the Bayesian game are well-defined.

The first-order conditions for expected utility maximization are that for each $i$, and given the strategy profile $f_{-i}$ of the other individuals and type $t \in \mathcal{T}$,

$$
\psi_{i}+\phi_{i} \sum_{j} a_{i j} \mathrm{E}\left(f_{j}\left(\psi_{j}, z_{j}\right) \mid x, z_{i}\right)-\left(1+\phi_{i}\right) \omega_{i}=0 .
$$


Since the problem is concave in $\omega_{i}$, the first-order conditions are sufficient.

Define the operator $T: \mathcal{F} \rightarrow \mathcal{F}$ such that

$$
(T f)_{i}\left(\psi_{i}, z_{i}\right)=\frac{1}{1+\phi_{i}} \psi_{i}+\frac{\phi_{i}}{1+\phi_{i}} \sum_{j} a_{i j} \mathrm{E}\left(f_{j}\left(\psi_{j}, z_{j}\right) \mid x, z_{i}\right) .
$$

A fixed point of $T$ satisfies the first-order condition for all $i, \psi_{i}$ and $z_{i}$; thus it will be a Bayes-Nash equilibrium profile. Assumption T.1 and a computation shows that this map is a contraction in the norm topology with contraction constant $\phi=$ $\max _{i} \phi_{i} /\left(1+\phi_{i}\right)$, and so a fixed point exists, and is unique. The fixed-point strategy profile satisfies the sufficient first-order optimality conditions, and so it is a Bayes-Nash equilibrium.

Any strategy profile can be written in the form

$$
f(x, z)=\Phi(I-\hat{\Phi} A)^{-1}(\gamma I+\delta C) x+g(x, z) .
$$

where $g_{i}(x, z)$ depends on $z$ through $z_{i}$ alone. Apply the operator $T$ to see that $f$ will be an equilibrium if and only if $g(x, z)$ satisfies, for each $i$,

$$
g_{i}(x, z)=\frac{1}{1+\phi_{i}} z_{i}+\frac{\phi_{i}}{1+\phi_{i}} \sum_{j} a_{i j} \mathrm{E}\left(g_{j}\left(x, z_{j}\right) \mid x, z_{i}\right) .
$$

Thus each $g_{i}$ depends upon $z$ only through $z_{i}$. From now on we take the arguments of each $g_{i}$ to be $x$ and $z_{i}$. Take

$$
\mu(x, z)_{i}=\sum_{j} a_{i j} \mathrm{E}\left(g_{j}\left(x, z_{j}\right) \mid x, z_{i}\right) .
$$

This proves the general characterization of equilibrium strategy profiles.

For the characterizations of the $g_{i}\left(x, z_{i}\right)$, define the operator $T_{g}$ such that

$$
\left(T_{g} h\right)_{i}=\frac{1}{1+\phi_{i}} z_{i}+\frac{\phi_{i}}{1+\phi_{i}} \sum_{j} a_{i j} \mathrm{E}\left(h_{j}\left(x, z_{j}\right) \mid x, z_{i}\right) .
$$

This operator too is a contraction on $L_{\rho}^{2}$, and so it has a unique fixed point, which is clearly $g$. The characterizations are proven by showing that the different assumptions imply that sets of $g$ with given properties are invariant under $T$, and so the fixed point must be in this set.

To prove the second claim, suppose now that $x$ and $z$ are independent. Then for any function $h_{j}: z_{j} \mapsto \mathbf{R}$,

$$
\mathrm{E}\left(h_{j}\left(z_{j}\right) \mid x, z_{i}\right)=\mathrm{E}\left(h_{j}\left(z_{j}\right) \mid z_{i}\right) .
$$

Consequently, the set of functions $h:(z) \mapsto \mathbf{R}^{\mathbf{N}}$ is invariant under $T_{g}$. Thus each $g_{i}$ depends only on $z_{i}$. 
For the third claim, observe that if the private types are independent, then if $h_{i}\left(x, z_{i}\right)$ is of the form $\left(1+\phi_{i}\right)^{-1} z_{i}+\mu_{i}(x)$, then

$$
T_{g}(h)_{i}(x, z)=\frac{1}{1+\phi_{i}} z_{i}+\frac{\phi}{1+\phi_{i}} \sum_{j} a_{i j}\left(\frac{1}{1+\phi_{j}} \mathrm{E}\left(z_{j} \mid x\right)+\mu_{j}(x)\right),
$$

since $\mathrm{E}\left(z_{j} \mid x, z_{i}\right)=\mathrm{E}\left(z_{j} \mid x\right)$. The sum over $j$ is a function only of $x$, and so the set of all functions of this form is invariant under $T_{g}$. Thus the fixed point has this property too. This proves theorem A1.

To complete the proof of theorem 1, observe that if for all $i$ and $j, \phi_{i}=\phi_{j}$, and if the $z_{i}$ are independent of each other and of $x$, the fixed point of $T_{g}$ can be computed directly, and gives equation $(2 a)$.

The remainder term $\mu(x, z)$ has to do with higher-order beliefs. Suppose, to simplify the exposition, that all the $\phi_{i}$ are identical. Equation (25) contains a recursion, and by iterating it, one sees that

$$
\begin{aligned}
\mu_{i}\left(x, z_{i}\right)=\frac{1}{1+\phi} & \left(\frac{\phi}{1+\phi} \sum_{i^{\prime}} a_{i i^{\prime}} \mathrm{E}\left(z_{i^{\prime}} \mid x, z_{i}\right)\right. \\
& \left.+\left(\frac{\phi}{1+\phi}\right)^{2} \sum_{i^{\prime}} \sum_{i^{\prime \prime}} a_{i i^{\prime}} a_{i^{\prime} i^{\prime \prime}} \mathrm{E}\left(\mathrm{E}\left(z_{i^{\prime \prime}} \mid x, z_{i^{\prime}}\right) \mid x, z_{i}\right)+\cdots\right)
\end{aligned}
$$

The second term contains expressions whose meanings are, " $i$ 's expectation of $j$ 's expectation of $z_{k} \ldots$ ".

Now we take up identification questions. We have assumed for convenience (E.5) that $\gamma$ and $\delta$ are not both 0 . Lemma 1 settles the question of identification when the true $\gamma$ and $\delta$ are both 0 .

Lemma 1. Assume T.1-3 and E.1-5. The set of parameters $\{(\gamma, \delta, \phi): \gamma=$ $\delta=0, \phi \geq 0\}$ is weakly identified from the conditional mean of $\omega$. No parameter vector $(0,0, \phi)$ is identified.

Proof of lemma 1. Let $\gamma^{*}, \delta^{*}$ and $\phi^{*}$ denote the true values of the un-starred parameters. If $\gamma^{*}=\delta^{*}=0$, then $B(s)=0$. If $B(s)=0$, then since $B_{\phi}(s)$ is non-singular, $\gamma^{*} I+\delta^{*} C=0$. The sociomatrix $C$ has some positive off-diagonal element (E.3), so the unique solution to the equation $\gamma I+\delta C=0$ is $\gamma=\gamma^{*}$ and $\delta=\delta^{*}$.

If $\gamma^{*}=\delta^{*}=0$, then $B(s)=0$, and $\phi$ affects $\omega$ only through its effect on $\varepsilon$. Since $\mathrm{E}(\varepsilon \mid x)=0, \mathrm{E}(\omega \mid x)$ is independent of the parameter $\phi$.

Proof of Theorem 2. Identification of $\mu$ follows from E.1 and equation (5). E $(\omega \mid x)$ is an affine function whose behavior on an open set is observed. Of course $\mathrm{E}(\omega \mid x)$ is identified; this, the spanning assumption E.1 and the orthogonality 
assumption E.4 identify $B(s)$. Since $C$ is stochastic, $B(s) e=(\gamma+\delta) B_{\phi}(s) e=$ $(\gamma+\delta) e$.

If $\mathrm{E}(\omega \mid x)$ is independent of $x$, then $B(s)=0$. Since $B_{\phi}(s)$ is always nonsingular, $\gamma I+\delta C=0$. E.3 implies that $C$ is not a multiple of $I$, so $\gamma=\delta=0$. Conversely, if $\gamma=\delta=0$, then $B(s)=0$ and $\omega$ is independent of $x$.

If for all $i, \mathrm{E}\left(\omega_{i} \mid x\right)=\mathrm{E}\left(\omega_{i} \mid x_{i}\right)$, then $B(s)=\alpha I$, and $\alpha$ is identified. It follows that

$$
\left(\gamma-\frac{\alpha}{1+\phi}\right) I+\delta C+\frac{\alpha \phi}{(1+\phi)^{2}} A=0 .
$$

If the a priori hypothesis is satisfied, T.1 implies that $I, C$ and $A$ are linearly independent, and it follows that $\delta=\phi=0$ and $\gamma=\alpha$. The converse is obvious.

Proof of Theorem 3. Part $i$ : Suppose that $s^{\prime}$ and $s^{\prime \prime}$ are two structures in $\mathcal{M}$ that are identical except perhaps for the values they assign to the parameters $\gamma, \delta$ and $\phi$. Then $B\left(s^{\prime}\right)=B\left(s^{\prime \prime}\right)$ if and only if

$$
B_{\phi}\left(s^{\prime}\right)\left(\gamma^{\prime} I+\delta^{\prime} C\right)=B_{\phi}\left(s^{\prime \prime}\right)\left(\gamma^{\prime \prime} I+\delta^{\prime \prime} C\right) .
$$

Multiply this out:

$$
\left(\left(1+\phi^{\prime \prime}\right) I-\phi^{\prime \prime} A\right)\left(\gamma^{\prime} I+\delta^{\prime} C\right)=\left(\left(1+\phi^{\prime}\right) I-\phi^{\prime} A\right)\left(\gamma^{\prime \prime} I+\delta^{\prime \prime} C\right),
$$

since $B_{\phi}\left(s^{\prime}\right), B_{\phi}\left(s^{\prime \prime}\right)$, and their inverses, all being power series in $A$, all commute. Consequently, $B\left(s^{\prime}\right)=B\left(s^{\prime \prime}\right)$ if and only if

$$
\begin{aligned}
\left(\left(1+\phi^{\prime \prime}\right) \gamma^{\prime}-\left(1+\phi^{\prime}\right) \gamma^{\prime \prime}\right) & I+\left(\left(1+\phi^{\prime \prime}\right) \delta^{\prime}-\left(1+\phi^{\prime}\right) \delta^{\prime \prime}\right) C \\
& +\left(\phi^{\prime} \gamma^{\prime \prime}-\phi^{\prime \prime} \gamma^{\prime}\right) A+\left(\phi^{\prime} \delta^{\prime \prime}-\phi^{\prime \prime} \delta^{\prime}\right) A C=0
\end{aligned}
$$

Independence of $I, A, C$ and $A C$ implies that each coefficient is 0 . Now enumerate cases. First, suppose that one of $\phi^{\prime}$ and $\phi^{\prime \prime}$ is 0 . Wlog assume $\phi^{\prime}=0$. Looking at the coefficients of $A$ and $A C$, independence implies that either $\phi^{\prime \prime}=0$ or $\gamma^{\prime}=\delta^{\prime}=0$. The second possibility has been dispensed with in lemma 1 , so conclude that $\phi^{\prime \prime}=0$. It follows immediately from the independence hypothesis that $\gamma^{\prime}=\gamma^{\prime \prime}$ and $\delta^{\prime}=\delta^{\prime \prime}$.

Next suppose that neither $\phi^{\prime}$ nor $\phi^{\prime \prime}$ is 0 . If either $\gamma^{\prime}$ or $\gamma^{\prime \prime}=0$, then the other must be 0 for the coefficient of $A$ to equal 0 . Similarly, if either $\delta^{\prime}$ or $\delta^{\prime \prime}=0$, then the other must be 0 for the coefficient of $A C$ to equal 0 . Suppose that $\gamma^{\prime} \neq 0$. Then from the coefficients for $I$ and $A$ deduce that $\left(1+\phi^{\prime \prime}\right) /\left(1+\phi^{\prime}\right)=\phi^{\prime \prime} / \phi^{\prime}$, and so $\phi^{\prime}=\phi^{\prime \prime}$. Conclude from the coefficient of $I$ that $\gamma^{\prime}=\gamma^{\prime \prime}$. A similar argument applies to the coefficient of $C$ if $\delta^{\prime} \neq 0$.

To establish the other direction, suppose that $\alpha I+\beta C+\theta A+\tau A C=0$ for $\alpha, \beta, \theta, \tau$ not all 0 . The parameters of the structures $s^{\prime}$ and $s^{\prime \prime}$ will satisfy equation 
(27) if and only if they satisfy the following matrix equation:

$$
\left(\begin{array}{cccc}
1+\phi^{\prime \prime} & -1-\phi^{\prime} & 0 & 0 \\
-\phi^{\prime \prime} & \phi^{\prime} & 0 & 0 \\
0 & 0 & 1+\phi^{\prime \prime} & -1-\phi^{\prime} \\
0 & 0 & -\phi^{\prime \prime} & \phi^{\prime}
\end{array}\right)\left(\begin{array}{c}
\gamma^{\prime} \\
\gamma^{\prime \prime} \\
\delta^{\prime} \\
\delta^{\prime \prime}
\end{array}\right)=\left(\begin{array}{l}
\alpha \\
\theta \\
\beta \\
\tau
\end{array}\right)
$$

The determinant of the matrix is $\left(\phi^{\prime}-\phi^{\prime \prime}\right)^{2}$. Choose any $\phi^{\prime} \neq \phi^{\prime \prime}$, both nonnegative. Then there will be parameters $\gamma^{\prime}, \gamma^{\prime \prime}, \delta^{\prime}$ and $\delta^{\prime \prime}$ which solve the equation. Consequently, for these parameter values $B\left(s^{\prime}\right)=B\left(s^{\prime \prime}\right)$, and the two parameter vectors cannot be distinguished.

To complete the proof we must show that even when $\phi$ cannot be identified, if either one of $\gamma$ and $\delta$ is identified, so is the other. A necessary and sufficient condition for $\gamma^{\prime}=\gamma^{\prime \prime}$ is that $\theta=-\alpha$. Thus $\alpha(I-A)+(\beta I+\tau A) C=0$. For each peer- effects component $b$ (a component is a maximal connected subnetwork), denote by $\pi_{b}$ the left Perron eigenvector of $A_{b}$ and let $C_{b}$ denote the $|b| \times N$ submatrix of $C$ consisting of the rows of $b$ and all columns. Then $(\beta+$ $\tau) \pi_{b} C_{b}=0$ for all components $b$. Each $\pi_{b}$ is strictly positive, and some $C_{b}$ does not equal 0 , so conclude that $\beta=-\tau$. This implies from the equation system that $\delta^{\prime}=\delta^{\prime \prime}$. Conversely, if $\delta^{\prime}=\delta^{\prime \prime}$, then $\beta=-\tau$, and so $(\alpha+\theta) \pi_{b}=0$. hence $\theta=-\alpha$.

Part ii. $B\left(s^{\prime}\right)=B\left(s^{\prime \prime}\right)$ if and only if

$$
\left(\left(1+\phi^{\prime \prime}\right) \gamma^{\prime}-\left(1+\phi^{\prime}\right) \gamma^{\prime \prime}\right) I+\left(\delta^{\prime}-\delta^{\prime \prime}\right) C+\left(\phi^{\prime} \gamma^{\prime \prime}-\phi^{\prime \prime} \gamma^{\prime}\right) A=0 .
$$

Suppose that $I, A$ and $C$ are linearly independent. Then each coefficient in equation (28) is 0 . In particular, the coefficient on $C$ is 0 , and so $\delta^{\prime}=\delta^{\prime \prime} ; \delta$ is identified.

The remaining coefficients are 0 if and only if

$$
\begin{gathered}
\phi^{\prime} \gamma^{\prime \prime}=\phi^{\prime \prime} \gamma^{\prime}, \text { and } \\
\left(1+\phi^{\prime}\right) \gamma^{\prime \prime}=\left(1+\phi^{\prime \prime}\right) \gamma^{\prime} .
\end{gathered}
$$

We need to show that $\gamma^{\prime}=\gamma^{\prime \prime}$ and $\phi^{\prime}=\phi^{\prime \prime}$. Observe first that, from the second equation, $\gamma^{\prime \prime}=0$ if and only if $\gamma^{\prime}=0$. Next observe that if $\gamma^{\prime} \neq 0, \phi^{\prime} /\left(1+\phi^{\prime}\right)=$ $\phi^{\prime \prime} /\left(1+\phi^{\prime \prime}\right)$. Thus $\phi^{\prime}=\phi^{\prime \prime}$, and it follows that $\gamma^{\prime}=\gamma^{\prime \prime}$.

For the converse, observe that if $\alpha I+\beta C+\theta A=0$ and any coefficient is not 0 , then $\beta \neq 0$ because no single coefficient can be non-zero, and if $\beta=0$ then $A$ would be a multiple of $I$, contradicting T.1. It follows from equation (28) that $\delta$ cannot be identified. Choose $\phi^{\prime} \neq \phi^{\prime \prime}$, and consider the equation

$$
\left(\begin{array}{cc}
1+\phi^{\prime \prime} & -\left(1+\phi^{\prime}\right) \\
-\phi^{\prime \prime} & \phi^{\prime}
\end{array}\right)\left(\begin{array}{l}
\gamma^{\prime} \\
\gamma^{\prime \prime}
\end{array}\right)=\left(\begin{array}{l}
a \\
c
\end{array}\right) \text {. }
$$

This equation says that the coefficient of $I$ is equation (28) equals $\alpha$ and the coefficient of $A$ in equation (28) equals $\theta$. The matrix is non-singular, and so there will be $\gamma^{\prime}$ and $\gamma^{\prime \prime}$ which solve it. Consequently, $\phi$ is not identified. Finally, We 
show by contradiction that $\gamma$ is not identified. if $\gamma$ is identified, then the equation system is solved, for all $\phi^{\prime} \neq \phi^{\prime \prime}$, by some $\gamma^{\prime}=\gamma^{\prime \prime}$. For this to be true, for all $\phi^{\prime} \neq \phi^{\prime \prime},\left(\phi^{\prime \prime}-\phi^{\prime}\right) \gamma^{\prime}=\alpha$ and $\left(\phi^{\prime}-\phi^{\prime \prime}\right) \gamma^{\prime}=\theta$, so $\theta=-\alpha$. If so, then $\alpha(I-A)+\beta C=0$. If the coefficient $\alpha$ equals 0 , then $\beta$ equals 0 as well, which is a contradiction, so $\alpha$ must not equal 0 . But $\alpha$ must equal 0 , because the matrix $\alpha(I-A)$ has both positive and negative elements (by T.1, the diagonal and offdiagonal elements have different signs), while the non-zero elements of $\beta C$ all have the same sign. This establishes the contradiction.

Proof of Corollary 1. The result follows directly from lemma 2.

Lemma 2. Suppose that $A$ is a peer-effects matrix with $N \geq 3$. Suppose $C$ is a contextual-effects sociomatrix such that for some peer-effects component $b$ with subgraph sociomatrix $C_{b b}$ :

(1) The invariant distribution $\pi_{b}$ of $A_{b}$ is not an eigenvector of $C_{b b}$.

(2) $C_{b b}$ has no eigenspace of dimension at least $|b|-1$, and

(3) $\operatorname{rank} C_{b b} \geq 2$.

Then the utility parameters are identified.

Proof of Lemma 2. It suffices to show that, generically, I, A, C and $A C$ are independent. If so, then $C \neq A C$, and the criterion of the first case of theorem 3 applies. So suppose that $\alpha I+\beta A+\theta C+\tau A C=0$. Post-multiply the sum by the vector of $1 \mathrm{~s}$, to conclude that $\alpha+\beta+\theta+\tau=0$.

Next, observe that for each pair of component $b$ of the peer-effects network,

$$
\alpha I+\beta A_{b}+\theta C_{b b}+\tau A_{b} C_{b b}=0,
$$

Let $\pi_{b}$ be a left Perron eigenvector for $A_{b}$. Then $(\alpha+\beta) \pi_{b}+(\theta+\tau) \pi_{b} C_{b b}=0$. By hypothesis, neither vector in the sum is 0 , and $\pi_{b}$ and $\pi_{b} C_{b b}$ are linearly independent, so $\alpha+\beta=\theta+\tau=0$.

Rewriting the linear combination of matrices, for all components $b$,

$$
\alpha\left(I-A_{b}\right)\left(I+\theta C_{b b}\right)=0 .
$$

Suppose that $\alpha \neq 0$. The null space of $I-A_{b}$ is spanned by $e$, so the range of $I+\theta C_{b b}$ is the vector space spanned by $e$. Consequently, $\theta \neq 0$. If rank $I+$ $\theta C_{b b}=0$, then $C_{b b}=-\theta^{-1} I$, which contradicts the hypothesis.

If rank $I+\theta C_{b b}$ is 1 , then the null space of $I+\theta C$ has rank equal to $|b|-1$, where $|b|$ is the number of nodes in the component. Hence $-\theta^{-1}$ is an eigenvalue of $C_{b b}$, of geometric multiplicity $|b|-1$. This too contradicts the hypothesis. Conclude that $\alpha=0$.

If $\alpha=0$, then $\theta\left(I-A_{b}\right) C_{b b}=0$. Again from the Perron-Frobenius Theorem, this can only hold if rank $C_{b b}=1$, which our hypothesis rules out. 
Lemma 3. $\quad i$. For all peer-effects sociomatrices $A$ containing a component $b$ of size $n^{b} \geq 3$, the set of contextual-effects sociomatrices $C$ for which $I$, $A, C$ and $A C$ are not independent is a closed-semi-algebraic set of lower dimension in $\mathcal{M}_{C}$.

ii. If $N \geq 3$ there is a closed semi-algebraic set $\mathcal{G}_{c} \subset \mathcal{M}_{C}$ which is the complement of a lower-dimensional in $\mathcal{M}_{C}$ such that for each $C$ in $\mathcal{G}_{C}$ the subset $S_{C}$ of $\mathcal{M}_{A}$ containing those peer-effects sociomatrices $A$ for which $I, A, C$ and $A C$ are not linearly independent is semi-algebraic, closed, and lower-dimensional in $\mathcal{M}_{A}$.

Proof of Lemma 3. The result follows immediately from lemma 2. Part $i$ is the corollary.

Proof of Corollary 2. Consider the equation system

$$
w I+x A+y C+z A C=0 .
$$

We will show that linear independence of all four matrices is the right condition to check, and then that the only solution to equation (29) is $w=x=y=z=$ 0 . The result then follows from theorem 3 . Let $q_{0}, \ldots, q_{n}$ be nodes such that i) $q_{0}=j$ and $q_{n}=i$;ii) each $q_{v}$ is linked to $q_{v-1}$ and $q_{v+1}$ (whenever they exist), by an edge in the peer-effects network and/or an edge in the contextual-effects network; and iii) this path is shortest possible among all such paths connecting $a$ and $z$. Denote by $\sim_{A}$ and $\sim_{C}$ peer- and contextual-effects edges. Thus we have $q_{0} \sim_{X_{0}} q_{1} \sim_{X_{1}} \cdots \sim_{X_{n-1}} q_{n}$, where each $X_{v}$ is either a $C$ or an $A$. For any particular $v$ it may be possible to take either $C$ or $A$ for $X_{v}$, but by hypothesis there is at least one $X_{v}$ which must be a $C$ and another which must be an $A$. Furthermore, minimality of the length implies that for all $w \neq v, q_{w} \neq q_{v}$, and that there is no $A$ or $C$ edge between $q_{v}$ and $q_{v+u}$ for $u \geq 2$.

Without loss of generality (run the chain backwards if necessary) there exists a triple $q_{v} \sim_{A} q_{w} \sim_{C} q_{u}$ where $u=w+1=v+2$. Then

$$
A C_{q_{v} q_{u}} \geq a_{q_{v} q_{w}} c_{q_{w} q_{u}}>0 \text { but } A_{q_{v} q_{u}}=C_{q_{v} q_{u}}=I_{q_{v} q_{u}}=0 \text {. }
$$

This shows two things: First, $I, A$ and $C$ are already known to be distinct by virtue of axioms T.1, E.2 and E.3. This calculation shows $A C$ to be distinct from these matrices. Thus, part i) of theorem 3 applies. Second, from the calculation infer that in any solution to equation (29), $z=0$.

Next, create a "C-maximal path" by choosing $X_{v}$ equal to $C$ wherever possible in the chain. By hypothesis, some $A$-edge must remain. Again without loss of generality choose a triple $q_{v} \sim_{A} q_{w} \sim_{C} q_{u}$ where $u=w+1=v+2$. Then $a_{q_{v} q_{w}}>0$ while $c_{q_{v} q_{w}}=I_{q_{v} q_{w}}=0$. Since $z=0$, this implies that $x=0$ in any solution to equation (29). Finally, create an " $A$-maximal path" and use the same argument to infer that $y=0$. If $x=y=z=0$, then $w=0$ and we are done. 
Proof of Corollary 3. Let $c$ denote a component of the contextual-effects network satisfying the hypotheses. Then $A C=C$, which is case ii) of theorem 3 . If $I, A$ and $C$ are linearly dependent, there are coefficients $\alpha, \beta$ and $\theta$ not all 0 such that $\alpha I+\beta A+\theta C=0$. The coefficient $\alpha$ is not 0 because $A$ is not a scalar multiple of $C$. The coefficient $\beta$ is not 0 because $C$ has off-diagonal positive elements, and the coefficient $\theta$ is not 0 because $A$ has diagonal elements all 0 (T.1). So $C$ is a linear combination of $I$ and $A$. But since all off-diagonal elements of $C$ are equal, the hypothesis on $A$ implies this cannot be the case.

The proof of theorem 4 is long, tedious, and without merit beyond its existence. It will be useful to rewrite the social interaction effects with the parameter $r=$ $\phi /(1+\phi)$, with $r \in[0,1)$.

Define $a(r)^{T}=(1-r) e^{T}(I-r A)^{-1}$, where $e$ is a vector of suitable length and $T$ denotes transpose. The effect of $r$ is isolated in the column-sum vector $a$. We need these facts:

Lemma 4. (a) For every sociomatrix $A$ which is not bistochastic, the map $r \mapsto$ $a(r)$ is an injection.

(b) For all $A$ and $r \in[0,1), a(r) \gg 0$.

(c) $\sum_{x} a_{x}(r)=N$.

Proof. We use the relationship $a^{T}=(1-r) e^{T}+r a^{T} A$ which is easily derived from the definition of $a$.

(a) If $a\left(r^{\prime}\right)=a\left(r^{\prime \prime}\right)=a$ for $\left.r^{\prime} \neq r^{\prime \prime}\right)$, then

$$
\left(r^{\prime \prime}-r^{\prime}\right) e^{T}+\left(r^{\prime}-r^{\prime \prime}\right) a^{T} A=0,
$$

so $a^{T} A=e^{T}$ and therefore $a=e$, and $A$ is bistochastic.

(b) Without loss of generality, suppose that $A$ is irreducible. (Otherwise consider each component of the peer-effects network $\mathcal{A}$ separately.) $(1-r) e^{T}(I-$ $r A)^{-1}$ equals $(1-r)\left(e+r e A+r^{2} e A^{2}+\cdots\right)$. This is the sum of nonnegative vectors, and some $e A^{T}$ is strictly positive.

(c) The row sums of $A$ are 1 .

Another technical lemma we need is this: Fix $N$, and let $S$ denote the set of all triples $(\alpha, r, C)$ which solve the equation system

$$
\alpha\left(a_{i}(r)-a_{j}(r)\right)-\beta \sum_{x \in V} a_{x}(r)\left(c_{x i}-c_{x j}\right)=0 \text { for } i \neq j,
$$

and let $S_{C} \subset \mathcal{M}_{C}$ denote its projection onto the set of all contextual-effects sociomatrices. 
Lemma 5. The set $S_{C}$ is closed and has dimension at most $2+(N-1)^{2}$, which is less than $\operatorname{dim} \mathcal{M}_{C}$ for $N \geq 3$.

The set of stochastic matrices had dimension $N(N-1)$, which exceeds $2+$ $(N-1)^{2}$ when $N \geq 3$. The proof involves facts about semi-algebraic sets sets defined by finite numbers of polynomial inequalities, which can be found, for instance, in Bochnak, Coste, and Roy (1987).

Proof of lemma 5. This system contains $N-1$ equations. According to lemma 4, $a(r) \gg 0$, and so the derivative with respect to $C$ of the left-hand side is surjective onto $\mathbf{R}^{\mathbf{N}-\mathbf{1}}$. Consequently the solution set is a semi-algebraic set of co-dimension $N-1$, which is to say, of dimension $2+(N-1)^{2}$. It is also compact. The projection of $S$ onto $S_{C}$ is compact and has dimension at most $2+(N-1)^{2}$ since semi-algebraic functions (projection, in this case) cannot increase the dimension of their domains.

Proof of Theorem 4. Since $\bar{x}^{g}$ is observed, $\sigma_{x}^{2}$ is identified. It is convenient to define, for the gth group,

$$
f^{g}\left(\gamma, \phi, \beta, A^{g}, C^{g}\right)=\frac{1}{n^{g}} e \cdot B_{\phi}^{g}\left(\gamma I+(\beta-\gamma) C^{g}\right)\left(\gamma I+(\beta-\gamma) C^{g T}\right) B_{\phi}^{g T} e^{T} .
$$

Then $v^{g}=f^{g}\left(\gamma, \phi, \beta, A^{g}, C^{g}\right)-f^{0}\left(\gamma, \phi, \beta, A^{0}, C^{0}\right)$.

Define $F=F^{1}, \ldots, F^{g}$ such that

$$
\begin{aligned}
F^{g}\left(\gamma^{\prime}, r^{\prime}, \gamma^{\prime \prime}, r^{\prime \prime}, \beta, A^{g}, C^{g}\right)= & f^{g}\left(\gamma^{\prime}, r^{\prime}, \beta, A^{g}, C^{g}\right)-f^{g}\left(\gamma^{\prime}, r^{\prime}, \beta, A^{0}, C^{0}\right)- \\
& f^{g}\left(\gamma^{\prime \prime}, r^{\prime \prime}, \beta, A^{g}, C^{g}\right)+f^{g}\left(\gamma^{\prime \prime}, r^{\prime \prime}, \beta, A^{0}, C^{0}\right)
\end{aligned}
$$

The domain of $F$ is taken to be $\mathbf{R}^{4} / \Delta^{2} \times \mathbf{R} \times M_{\mathcal{A}} \times M_{\mathcal{C}}$ where $\mathcal{A}$ and $\mathcal{C}$ denote the peer- and contextual-effects networks, respectively and the first set is that of all quadruples $\left(\gamma^{\prime}, r^{\prime}, \gamma^{\prime \prime}, r^{\prime \prime}\right)$ such that not both $\gamma^{\prime}=\gamma^{\prime \prime}$ and $r^{\prime}=r^{\prime \prime}$.

Fix the $A^{g}, C^{g}$ and $s$, and consider the equation

$$
F\left(\gamma^{\prime}, r^{\prime}, \gamma^{\prime \prime}, r^{\prime \prime}, \beta, A^{0}, \ldots, A^{g}, C^{0}, \ldots, C^{g}\right)=0 .
$$

Since $\beta$ is already identified, we need only to identify $\gamma$ and $r$. The statistic $\left(v^{1}, \ldots, v^{g}\right)$ does not distinguish $\gamma^{\prime}, r^{\prime}$ from $\gamma^{\prime \prime}, r^{\prime \prime}$ (given $\beta$ ) if and only if $\left(\gamma^{\prime}, r^{\prime}, \gamma^{\prime \prime}, r^{\prime \prime}\right)$ solves the equation, and $\left(\gamma^{\prime}, r^{\prime}\right) \neq\left(\gamma^{\prime \prime}, r^{\prime \prime}\right)$. Thus we must show that for generic $C^{1}, \ldots, C^{g}$,

$$
F\left(\gamma^{\prime}, r^{\prime}, \gamma^{\prime \prime}, r^{\prime \prime}, \beta, A^{0}, \ldots, A^{g}, C^{0}, \ldots, C^{g}\right)=0
$$

has no solution in $R^{4} / \Delta^{2}$.

We will show that if $F^{g}=0$, then $D_{C^{g}} F^{g}$ is surjective onto $\mathbf{R}$. If so, it follows that $D F_{C^{1}, \ldots, C^{g}}$ is surjective onto $\mathbf{R}^{\mathbf{g}}$. Consequently, 0 is a regular value of $F$, and we conclude from the transversality theorem that for almost all $C^{1}, \ldots, C^{g}$, 0 is a regular value of the map $F\left(\cdot, \beta, A^{1}, \ldots, A^{g}, C^{1}, \ldots, A^{g}\right): \mathbf{R}^{4} / \Delta^{2} \rightarrow \mathbf{R}^{\mathbf{g}}$. 
Because $F$ is semi-algebraic, the set of critical $C^{1}, \ldots, C^{g}$ for which this may fail is closed and lower-dimensional in $\mathcal{M}_{C}^{\prime}$. When the map has 0 as a regular value, the inverse image of 0 is a manifold of co-dimension $G$. For $G \geq 5$, this implies that the solution set in $\mathbf{R}^{4} / \Delta^{2}$ has negative dimension, that is, it is empty.

It remains only to show that if $F^{g}=0$, then $D_{C^{g}} F^{g}$ is surjective onto $\mathbf{R}$. Observe first that

$$
D_{C^{g}} F^{g}=D_{C^{g}} f^{g}\left(\gamma^{\prime}, r^{\prime}, \beta, A^{g}, C^{g}\right)-D_{C^{g}} f^{g}\left(\gamma^{\prime \prime}, r^{\prime \prime}, \beta, A^{g}, C^{g}\right) .
$$

The derivative $D_{C^{g}} f^{g}$ is a linear map from the tangent space of $M_{\mathcal{C}}$ to $\mathbf{R}$. That tangent space is spanned by the set of all matrices $H_{k i j}$ whose vwth entry is 1 if $v=k$ and $w=i,-1$ if $v=k$ and $w=j$, and 0 otherwise. In words, $H_{k i j}$ shifts a little bit of $j$ 's influence on $k$ to $i$.

A calculation shows that

$$
\begin{aligned}
D_{C^{g}} f^{g} H_{k i j} & =a_{k}(r)(s-\gamma)\left(\gamma\left(a_{i}(r)-a_{j}(r)\right)+(s-\gamma) \sum_{x} a_{x}(r)\left(c_{i x}-c_{j x}\right)\right) \\
& \equiv a_{k}(r) \varrho_{i j}(\gamma, r)
\end{aligned}
$$

where $a=e B_{\phi}$. Thus if $D_{C^{g}} F^{g}$ is not surjective at $\left(\gamma^{\prime}, r^{\prime}, \gamma^{\prime \prime}, r^{\prime \prime}\right)$, then

$$
a_{k}\left(r^{\prime}\right) \varrho_{i j}\left(\gamma^{\prime}, r^{\prime}\right)=a_{k}\left(r^{\prime \prime}\right) \varrho_{i j}\left(\gamma^{\prime \prime}, r^{\prime \prime}\right) \text {. }
$$

First we show that for generic $C$ and all $\gamma$ and $r$, there is a pair $i \neq j$ such that $\varrho_{i j}(\gamma, r) \neq 0$. Suppose not. Since $\gamma \neq s$, for all $i, j$ pairs

$$
\gamma\left(a_{i}(r)-a_{j}(r)\right)=(\gamma-s) \sum_{x} a_{x}(r)\left(c_{x i}-c_{x j}\right) .
$$

Fix $j$. Then for all $i \neq j$, the equation system

$$
\alpha\left(a_{i}(r)-a_{j}(r)\right)=\sum_{x} a_{x}(r)\left(c_{x i}-c_{x j}\right)
$$

has a solution. From lemma 5 , the set of matrices $S_{C}$ for which this system has a solution is a closed and lower-dimensional subset of $\mathcal{M}_{C}^{\prime}$. So for $C \in S_{C}^{c}$, the equation system has no solution, and hence some $\varrho_{i j} \neq 0$.

Now suppose that $D_{C^{g}} F^{g}$ is not surjective at $\left(\gamma^{\prime}, r^{\prime}, \gamma^{\prime \prime}, r^{\prime \prime}\right)$, so that equation (30) holds. Lemma 4 states that the sum over $u$ of $a_{k}(r)$ is $N$, independent of $r$. Consequently, summing over $u$ in equation (30), we see that for all $i$ and $j$, $\varrho_{i j}\left(\gamma^{\prime}, r^{\prime}\right)=\varrho_{i j}\left(\gamma^{\prime \prime}, r^{\prime \prime}\right)$. Since for at least one $i, j$ pair, $\varrho_{i j}\left(\gamma^{\prime}, r^{\prime}\right) \neq 0$, it follows that for all $u, a\left(r^{\prime}\right)=a\left(r^{\prime \prime}\right)$. Conclude from lemma 4 that $r^{\prime}=r^{\prime \prime}$. Thus $D_{C^{g}} F^{g}$ can only fail to be surjective at points $\left(\gamma^{\prime}, r^{\prime}, \gamma^{\prime \prime}, r^{\prime}\right)$.

A calculation now shows that for all $i \neq j$,

$$
\left(s-\gamma^{\prime}-\gamma^{\prime \prime}\right)\left(a_{i}\left(r^{\prime}\right)-a_{j}\left(r^{\prime}\right)\right)=\left(2 s-\gamma^{\prime}-\gamma^{\prime \prime}\right) \sum_{x} a_{x}\left(r^{\prime}\right)\left(c_{x i}-c_{x j}\right),
$$


and by hypothesis $\gamma^{\prime} \neq \gamma^{\prime \prime}$. If $s \neq 0$, then at least one of $s-\gamma^{\prime}-\gamma^{\prime \prime}$ and $2 s-\gamma^{\prime}-\gamma^{\prime \prime}$ must not be 0 . That is, the equation system

$$
\alpha\left(a_{i}\left(r^{\prime}\right)-a_{j}\left(r^{\prime}\right)\right)=\beta \sum_{x} a_{x}\left(r^{\prime}\right)\left(c_{x i}-c_{x j}\right)
$$

has a solution $\left(\alpha, \beta, r^{\prime}, C\right)$ has a solution with not both $\alpha=\beta=0$. It cannot be the case that $\beta=0$, for if so, then $\alpha \neq 0$, and $a_{i}\left(r^{\prime}\right)=a_{j}\left(r^{\prime}\right)$ for all $i$ and $j$. But if this were the case, then $a\left(r^{\prime}\right)=e$ and it follows that $r^{\prime}=0$ or that $A$ is bistochastic. We have ruled out both cases by assumption. Since $\beta \neq 0$, it follows that if $D_{C^{g}} F^{g}$ is not surjective, then

$$
\alpha\left(a_{i}\left(r^{\prime}\right)-a_{j}\left(r^{\prime}\right)\right)=\sum_{x} a_{x}\left(r^{\prime}\right)\left(c_{x i}-c_{x j}\right),
$$

and again from lemma 5 , this can only happen for $C^{g} \in S_{C}$.

If $-\gamma^{*} / \delta^{*}$ is not an eigenvalue of $C$, then $B$ will be non-singular, and $\gamma^{*}, \delta^{*}$ and $\phi^{*}$ solve the equation

$$
(1+\phi) I-\phi A=\gamma B^{-1}+\delta C B^{-1} .
$$

This fact is used in the proofs of theorems 5 and 6 below.

Proof of Theorem 5. Axiom E.2 and the hypothesis imply that both $a_{j i}$ and $a_{i j}$ are 0 , so we have two locations with 0 's in the peer-effects sociomatrix. Let $\gamma^{*}, \delta^{*}$ and $\phi^{*}$ denote the true parameter values, and recall that $B=B(s)$ is identified (by theorem 2). Since it is nonsingular, they solve equation (31).

Although we do not know the matrix $A$, we do know that both the $j i$ and the $i j$ terms are 0 . We also know that $\beta^{*}=\delta^{*}+\gamma^{*}$ is identified (by theorem 2). Thus the following two-by-two equation system in $\gamma$ and $\delta$ has as one solution $\delta=\delta^{*}$ and $\gamma=\gamma^{*}$.

$$
\begin{aligned}
\gamma B_{j i}^{-1}+\delta \sum_{k} c_{j k} B_{k i}^{-1} & =0 \\
\gamma+\delta & =\beta^{*}
\end{aligned}
$$

Simplifying,

$$
\gamma B_{j i}^{-1}+\left(\beta^{*}-\gamma\right) \sum_{k} c_{j k} B_{k i}^{-1}=0 .
$$

The system is degenerate if and only if $B_{j i}^{-1}=\sum_{k} c_{j k} B_{k i}^{-1}=0$. When $B_{j i}^{-1} \neq 0$, equation (32) can be solved for $\gamma^{*}$, and since $\beta^{*}$, this gives $\delta^{*}$. Finally, identify $\phi$ from the diagonal of equation (31) - the equation

$$
1+\phi=\gamma^{*} B_{j j}^{-1}+\delta^{*} c_{j k} B_{k j}^{-1}
$$

has $\phi^{*}$ as its unique solution. 
Proof of theorem 6. Let $\gamma^{*}, \delta^{*}$ and $\phi^{*}$ denote the true utility parameter values. Since $A$ has 0 's on the diagonal, the right has side of equation (31) has identical diagonal elements when $\gamma=\gamma^{*}$ and $\delta=\delta^{*}$. Consider the equation system

$$
\gamma\left(B_{i i}^{-1}-B_{11}^{-1}\right)+\delta\left(B^{-1} C_{i i}-B^{-1} C_{11}\right)=0,
$$

for $i=2, \ldots, N$, with unknowns $\gamma$ and $\delta$. The system has $\gamma=\gamma^{*}$ and $\delta=\delta^{*}$ as a solution. The coefficients of this system are either observable or known a priori . This system has rank at most 1 . Obviously the rank cannot be more than 2 . If it is rank 2 , the unique solution would be $\gamma=\delta=0$, contradicting the hypothesis.

There are two possibilities, then: The system (33) has rank 0 or rank 1 . If the system has rank 0 , then in particular $\gamma=1$ and $\delta=0$ solve the system. Hence all the diagonal elements of $B^{-1}$ are identical. A derivative calculation and a transversality argument shows that for the complement of a closed, lowerdimensional subset of $\mathcal{M}_{A}$, however, the system has rank 1 .

If the system (33) has rank 1 , then the solution set is either $\delta=0$ or $\gamma=z \delta$ for some $z \neq 0$. In the first case, we have $\delta=\delta^{*}$, and so this case can only arise when $\delta^{*}=0$ and $\gamma^{*}=\beta^{*}$. Since $\beta^{*}$ is identified, we can infer $\gamma=\gamma^{*}=\beta^{*}$. In the second case, $\delta^{*}$ and $\gamma^{*}$ solve

$$
\begin{aligned}
\gamma+\delta & =\beta^{*} \\
\gamma-z \delta & =0 .
\end{aligned}
$$

Notice that $\beta^{*}$ cannot equal 0 by assumption - otherwise $-\gamma^{*} / \delta^{*}$ is an eigenvalue of $C$. If $z=-1$ this equation system is inconsistent, which cannot be the case. If $z \neq-1$, then it has a unique solution, which must be $\gamma=\gamma^{*}$ and $\delta=\delta^{*}$.

Proof of Corollary 4. Once $\phi, \gamma$ and $\delta$ are identified, $A$ is recovered from equation (31).

Proof of theorem 7. First we show that the conditions of the theorem are sufficient and necessary, respectively, for identifying the parameter vectors $\pi_{1}, \pi_{2}$ and $\pi_{3}$. Expand equation (19):

$$
\begin{aligned}
\omega_{i}=\pi_{1} x_{i}+\sum_{\substack{j \sim c^{i} i \\
j \neq i}} \pi_{2 j} x_{j} \\
\\
\quad+\sum_{k \sim A_{A} i} \pi_{3 k}\left(\sum_{\substack{j \sim c^{i} i \\
j \neq i}} b_{k j} x_{j}+b_{k i} x_{i}+\sum_{j \varkappa_{c} i} b_{k j} x_{j}\right)+\frac{1}{1+\phi} \varepsilon_{i}
\end{aligned}
$$

Reorder the elements of $B$, and partition it as follows: 


$$
\begin{aligned}
& j \sim_{C} i \quad j \nsim_{C} i \\
& B=\begin{array}{l}
i, j \sim_{A} i \\
j \varkappa_{A} i
\end{array}\left(\begin{array}{cccc}
b_{11} & \ldots & \ldots & b_{1 N} \\
& \vdots & \vdots & \\
b_{N 1} & \ldots & \ldots & b_{N N}
\end{array}\right)=\left(\begin{array}{ll}
\mathrm{B}_{A C} & \mathrm{~B}_{A \neg C} \\
\mathrm{~B}_{\neg A C} & \mathrm{~B}_{\neg A \neg C}
\end{array}\right) .
\end{aligned}
$$

Then the first term on the second line of equation (34) is

$$
\begin{aligned}
\left(\begin{array}{ll}
\pi_{3} & 0
\end{array}\right)\left(\begin{array}{ll}
\mathrm{B}_{A C} & \mathrm{~B}_{A \neg C} \\
\mathrm{~B}_{\neg A C} & \mathrm{~B}_{\neg A \neg C}
\end{array}\right)\left(\begin{array}{l}
x_{j \sim C^{i}} \\
x_{j \sim_{C} i}
\end{array}\right) & \\
= & =\pi_{3} \mathrm{~B}_{A C} x_{j \sim \sim_{C} i}+\pi_{3} \mathrm{~B}_{A \neg C} x_{j \varkappa_{C} i} .
\end{aligned}
$$

Identification of the parameters requires that the dimension of the set

$$
\left\{x_{i},\left(x_{j}\right)_{\substack{j \sim c i \\ j \neq i}}\left(x_{j}\right)_{\substack{j \sim c i \\ j \neq i}}: x \in \operatorname{supp}_{x} \rho\right\}
$$

be at least as big as the number of parameters to be estimated, where $\operatorname{supp}_{x} \rho$ denotes the support of the marginal distribution of $x$. The necessary condition follows from the fact that $N$ is an upper bound on this dimension. The sufficient condition comes from observing that the dimension of this set is $1+\#\left\{j: j \sim_{C}\right.$ $i, j \neq i\}+\operatorname{dim} \operatorname{rank} B_{A \neg C}$. and the needed dimension is $1+\#\left\{j: j \sim_{C} i, j \neq\right.$ $i\}+\#\left\{j: j \sim_{A} i\right\}$.

Next we show how to recover the parameters from the $\pi_{i} \cdot \sum_{j} \pi_{3 j}=(\phi /(1+$ $\phi) \sum_{j} a_{i j}$. Since that sum is $1, \phi$ is identified. With $\phi$ identified, each $\pi_{3 j}$ identifies $a_{i j}$. If any $\pi_{2 j}=0$ then $\delta=0$ and $\gamma$ is identified from $\beta$. Otherwise, $\pi_{2 k} / \pi_{2 j}=$ $c_{i u} / c_{i j}$. Since $\phi$ is identified, the sum $\sum_{j} \pi_{2 j}=\delta\left(1-c_{i i}\right)$. This, $\pi_{1}$ and $\beta$ deliver the one-dimensional curve described in the theorem. Finally, it is quick to check that failure to identify any $\pi_{i j}$ causes a consequent drop in information: Of $\rho$ and $a_{i}$ if a component of $\pi_{3}$ is unidentified, $c_{i k} / c_{i j}$ for some pair $(k, j)$ if one of those components of $\pi_{2}$ are not identified, and the curve containing $\gamma$ and $\delta$ is not identified if a component of $\pi_{2}$ or $\pi_{1}$ is not identified.

Proof of Theorem 8. Let $\hat{x}^{g}$ denote the mean of the $x_{j}$ for $j \in g$, including individual $i$. Equations (20) become

$$
\begin{aligned}
\mathrm{E}\left(\bar{\omega} \mid x_{i}, \bar{x}^{g}\right) & =\mu_{g}+b_{g} \hat{x}^{g}+b_{g i} x_{i} \\
\mathrm{E}\left(\omega_{i} \mid x_{i}, \bar{x}^{g}\right) & =\mu_{i}+b_{-i} \hat{x}^{g}+b_{i i} x_{i}
\end{aligned}
$$

The four coefficients are identified, and the problem is to determine the values of the utility parameters from these four values without knowing $A$. Observe that $b_{-i}+b_{i i}=\gamma+\delta$, a row-sum of $B$. Thus $\gamma+\delta$ is identified. Furthermore, $b_{g}+b_{g i}=(N-1)(\gamma+\delta)$. Consequently there are only three independent values among these four coefficients. For fixed $C$, let $F_{C}$ denote the map that takes quadruples $(\gamma, \delta, \phi, A)$ to triples $\left(b_{i i}, b_{-i}, b_{g i}\right)$ with the given $C$ matrix. This map is smooth, and so the implicit function theorem can be used to study solutions of the equation

$$
F_{C}(\gamma, \delta, \phi, A)=\left(b_{i i}, b_{-i}, b_{g i}\right)
$$


It will be convenient to take $i=1$ for the calculations, to reparametrize with $r=$ $\phi /(1+\phi)$, and to work with the map $G_{V}:(\gamma, \delta, r, \epsilon): \mapsto\left(b_{11}, \sum_{k} b_{k 1}, \sum_{i} b_{1 i}\right)$. This function is a non-singular linear transformation of $F_{C}$, and so we can identify utility parameters from $b_{i i}, b_{g i}$ and $b_{-i}$ if and only if we can identify them from these sums of elements of $B$ as well.

We will show that there is a particular direction $H$ for a perturbation of peereffects matrices $A$ such that for generic $A$ and any $\phi>0$, the derivative of the map $(\gamma, \delta, \phi, \epsilon) \mapsto G_{C}(\gamma, \delta, \phi, A+\epsilon H)$ has full-rank at $(\gamma, \delta, \phi, 0)$ and the partial derivative $\partial_{\epsilon} \neq 0$. Choose now $\gamma^{*}, \delta^{*}$ and $\phi^{*}$, and an $A$ for which the preceding statement holds, and denote the corresponding statistics $\left(s_{1}, s_{2}, s_{3}\right) \equiv$ $\left(b_{11}^{*}, \sum_{k} b_{k 1}^{*}, \sum_{i} b_{1 i}^{*}\right)$. The derivative map is surjective in a neighborhood $J \times I$ of $\left(\gamma^{*}, \delta^{*}, \phi^{*}, 0\right)$ where $I$ is an open interval around $\varepsilon=0$ and $J$ is an open rectangle in $\mathbf{R}^{3}$ containing $\left(\gamma *, \delta^{*}, r^{*}\right)$, and so the intersection of the inverse image of $\left(b_{11}^{*}, \sum_{k} b_{k 1}^{*}, \sum_{i} b_{1 i}^{*}\right)$ with $J \times I$ is a manifold of dimension 1 . In fact, we show that $J \times I$, the partial derivative $\partial_{\gamma \delta \epsilon} G_{C}: \mathbf{R}^{3} \rightarrow \mathbf{R}^{3}$ is surjective. This immediately implies that $r$ is not identified. Suppose we parametrize the manifold locally in a neighborhood $\left(\gamma^{*}, \delta^{*}, \phi^{*}, 0\right)$ as $\psi(\lambda)=(\gamma(\lambda), \delta(\lambda), r(\lambda), \epsilon(\lambda))$ where $\psi(0)=\left(\gamma^{*}, \delta^{*}, \phi^{*}, 0\right)$. Suppose $D \psi(0)=\left(x_{\gamma}, x_{\delta}, x_{r}, x_{\epsilon}\right)$. Then $D \psi(0) \neq 0$, and

$$
0=\partial_{r} G_{C}(\psi(0)) \cdot x_{r}+\partial_{\gamma \delta \epsilon} G_{C}(\psi(0)) \cdot\left(x_{\gamma}, x_{\delta}, x_{\epsilon}\right) .
$$

To show that $r$ is not identified, it suffices to show that $x_{r} \neq 0$. Suppose $x_{r}=0$. Since $D \psi(0) \neq 0, x_{r}=0$ requires that $\left(x_{\gamma}, x_{\delta}, x_{\epsilon}\right) \neq 0$. If so, then $D G_{C}(\psi(0))$. $D \psi(0) \neq 0$, which is a contradiction.

Now we calculate. First observe that for generic $A$, there will exist $i$ and $j$ such that $b_{1 i} \neq b_{1 j}$, and $a_{1 i}, a_{1 j}>0$. Next, observe that $D_{A} B \cdot H=-r(1-$ $r A)^{-1} H B$, where $r=\phi /(1+\phi)$. Observe, too, that $s_{2}=\gamma+\delta$. To show that $D G_{C}\left(\gamma^{*}, \delta^{*}, \phi^{*}, A\right)$ is surjective, it suffices to show that $\partial_{\gamma \delta \epsilon} G_{C}\left(\gamma^{*}, \delta^{*}, \phi^{*}, A+\right.$ $\epsilon H)\left.\right|_{\epsilon=0}$ has rank 3. Choose $H$ to be the matrix $H_{k i j}$ where $i$ and $j$ are as above, $u \neq 1, h_{k i}=-h_{k j}=1$, and all other elements of $H$ are 0 . Computing,

$$
\begin{aligned}
& \partial_{\gamma \delta \epsilon} G_{C}\left(\gamma^{*}, \delta^{*}, \phi^{*}, A+0 H\right)= \\
& \left(\begin{array}{ccc}
\left(1-r^{*}\right) d_{1,1} & \left(1-r^{*}\right) \sum_{u} d_{1 u} c_{u 1} & -r^{*}\left(b_{i 1}-b_{j 1}\right) d_{1 k} \\
1 & 1 & 0 \\
\left(1-r^{*}\right) \sum_{v} d_{v 1} & \left(1-r^{*}\right) \sum_{v} \sum_{u} d_{v k} c_{u 1} & -r^{*}\left(b_{i 1}-b_{j 1}\right) \sum_{u} d_{u k}
\end{array}\right)
\end{aligned}
$$

where $d_{u l}$ is the $u l$ th element of $\left(I-r^{*} A\right)^{-1}$. Since $b_{i 1}-b_{j 1} \neq 0$ and $0<r^{*}<$ 1 , this matrix is non-singular if and only if the following matrix is non-singular:

$$
\left(\begin{array}{ccc}
d_{1,1} & \sum_{u} d_{1 u} c_{u 1} & d_{1 k} \\
1+\phi^{*} & 1+\phi^{*} & 0 \\
\sum_{v} d_{v 1} & \sum_{v} \sum_{u} d_{v k} c_{u 1} & \sum_{u} d_{u k}
\end{array}\right)
$$


Notice that $\delta^{*}$ and $\gamma^{*}$ have disappeared. For fixed $r^{*}$ and $C$ it is generic in $A$ that this matrix is non-singular.

Proof of Theorem 9. The following conditions are generic: $I, A, C$ and $A C$ are independent, and $I, A+A^{T}, C+C^{T}, A A^{T}$ and $C C^{T}$ are independent. We show that these independence conditions are sufficient for the conclusions of the theorem. is

Identification of $\gamma, \delta$ and $\phi$ comes from theorem 3. The covariance matrix of $\omega$

$$
\begin{aligned}
\Omega & =(1-r)^{2} \sigma_{\varepsilon}^{2} I+B_{u} u \cdot\left(B_{u} u\right)^{T} \\
& =(1-r)^{2} \sigma_{\varepsilon}^{2} I+(1-r)^{2} \sigma_{u}^{2}(I-r A)^{-1}\left(\gamma_{u} I+\delta_{u} C\right)\left(\gamma_{u} I+\delta_{u} C^{T}\right)\left(I-r A^{T}\right)^{-1},
\end{aligned}
$$

and so

$$
\begin{aligned}
\hat{\Omega} & \equiv(1-r)^{-2}(I-r A) \Omega\left(I-r A^{T}\right) \\
& =\sigma_{\epsilon}^{2}\left(I-r\left(A+A^{T}\right)+r^{2} A A^{T}\right)+\sigma_{u}^{2}\left(\gamma_{u}^{2} I+\gamma_{u} \delta_{u}\left(C+C^{T}\right)+\delta_{u}^{2} C C^{T}\right) \\
& =\left(\sigma_{\epsilon}^{2}+\sigma_{u}^{2} \gamma_{u}^{2}\right) I-\sigma_{\epsilon}^{2} r\left(A+A^{T}\right)+\sigma_{u}^{2} \gamma_{u} \delta_{u}\left(C+C^{T}\right)+\sigma_{\epsilon}^{2} r^{2} A A^{T}+\sigma_{u}^{2} \delta_{u}^{2} C C^{T} .
\end{aligned}
$$

Since $r$ is identified and $A$ is known a priori, $\hat{\Omega}$ is observable. Suppose now that $\sigma_{\epsilon}^{2}, \gamma_{u}$ and $\delta_{u}$, and $\sigma_{\epsilon}^{2^{\prime}}, \gamma_{u}^{\prime}$ and $\delta_{u}^{\prime}$ generate the same $\hat{\Omega}$. As in the proof of theorem 3 , independence implies here that there is a $z$ such that $z=\sigma_{u}^{2} / \sigma_{u}^{2^{\prime}}>0$, $\delta_{u}^{\prime 2}=z \delta_{u}^{2}, \gamma_{u}^{\prime} \delta_{u}^{\prime}=z \gamma_{u} \delta_{u}, \sigma_{\epsilon}^{2 \prime}=\sigma_{\epsilon}^{2}$, and finally, $\gamma^{\prime 2}=z \gamma^{2}$. This is enough to establish that $\left(\gamma^{\prime}, \delta^{\prime}\right)= \pm \sqrt{z}(\gamma, \delta)$. 